\title{
ANALYSIS OF GRADIENT FLOW OF A REGULARIZED MUMFORD-SHAH FUNCTIONAL FOR IMAGE SEGMENTATION AND IMAGE INPAINTING
}

\author{
Xiaobing Feng ${ }^{1}$ And Andreas Prohl ${ }^{2}$
}

\begin{abstract}
This paper studies the gradient flow of a regularized Mumford-Shah functional proposed by Ambrosio and Tortorelli $(1990,1992)$ for image segmentation, and adopted by Esedoglu and Shen (2002) for image inpainting. It is shown that the gradient flow with $L^{2} \times L^{\infty}$ initial data possesses a global weak solution, and it has a unique global in time strong solution, which has at most finite number of point singularities in the space-time, when the initial data are in $H^{1} \times H^{1} \cap L^{\infty}$. A family of fully discrete approximation schemes using low order finite elements is proposed for the gradient flow. Convergence of a subsequence (resp. the whole sequence) of the numerical solutions to a weak solution (resp. the strong solution) of the gradient flow is established as the mesh sizes tend to zero, and optimal and suboptimal order error estimates, which depend on $\frac{1}{\varepsilon}$ and $\frac{1}{k_{\varepsilon}}$ only in low polynomial order, are derived for the proposed fully discrete schemes under the mesh relation $k=o\left(h^{\frac{1}{2}}\right)$. Numerical experiments are also presented to show effectiveness of the proposed numerical methods and to validate the theoretical analysis.
\end{abstract}

Mathematics Subject Classification. 35K55, 65M12, 65M15, 68U10, 94A08.

Received: June 10, 2003. Revised: September 5, 2003.

\section{INTRODUCTION}

Image segmentation in computer vision aims at automatic partitioning of a given image on $\Omega \subset \mathbf{R}^{N}(N=2,3)$ into regions where the gray-level function $u: \Omega \rightarrow \mathbf{R}$ is smooth, having only discontinuities across edges. A variational model was proposed by Mumford and Shah [31,32] to segment the image into as few and simple regions as possible and thus detect essential structures of the image. Following earlier discrete models proposed by D. Geman and S. Geman [24] and by Blake and Zisserman [7], they proposed to find:

$$
(v, \Gamma):=\underset{\substack{K \subset \Omega \text { closed } \\ u \in H^{1}(\Omega \backslash K)}}{\operatorname{argmin}} E(u, K),
$$

\footnotetext{
Keywords and phrases. Image segmentation and inpainting, Mumford-Shah model, elliptic approximation, gradient flow, a priori estimates, finite element method, error analysis.

1 Department of Mathematics, The University of Tennessee, Knoxville, TN 37996, USA.

2 Department of Mathematics, ETHZ, 8092 Zürich, Switzerland. e-mail: apr@math.ethz.ch
} 
for given $g \in L^{2}(\Omega), \alpha, \beta, \gamma \geq 0$. Where

$$
E(u, K):=\frac{\alpha}{2} \int_{\Omega \backslash K}|\nabla u|^{2} \mathrm{~d} x+\frac{1}{2} \int_{\Omega} \gamma|u-g|^{2} \mathrm{~d} x+\beta \mathcal{H}^{N-1}(K),
$$

and $\mathcal{H}^{N-1}(K)$ denotes the $(N-1)$-dimensional Hausdorff measure of $K$, which measures the "length" of the set $K$.

Heuristically, we expect solutions to this problem to be smooth and close to the image $g$ at places $x \notin \Gamma$, and $\Gamma$ constitutes edges of the image. To show existence of solutions to the above problem, a weak formulation was proposed by De Giorgi, Carriero and Leaci [17] by dropping the requirement that $K$ is a closed set, and to allow it to be the jump set of an $S B V$ (Special Bounded Variation) function $u$. So the idea was to find

$$
\left(\tilde{v}, S_{\tilde{v}}\right):=\underset{u \in S B V(\Omega)}{\operatorname{argmin}} \tilde{E}(u),
$$

where

$$
\tilde{E}(u):=\frac{\alpha}{2} \int_{\Omega \backslash S_{u}}|\nabla u|^{2} \mathrm{~d} x+\frac{1}{2} \int_{\Omega} \gamma|u-g|^{2} \mathrm{~d} x+\beta \mathcal{H}^{n-1}\left(S_{u}\right) .
$$

$S B V(\Omega)$ denotes the set of special bounded variation functions, and $S_{u}$ stands for the jump set of $u$, the complement of the set of all Lebesgue-points of $u$ in $\Omega$ (cf. [4]). The existence of solutions to problem (1.2) was established in [17], and $(v, \Gamma)=\left(\tilde{v}, \overline{S_{\tilde{v}}}\right)$ was proved for a large range of applications.

Since the above variational problems require the computation of geometrical properties of the unknown set of discontinuity boundaries, this results in considerable difficulties to numerically compute the solutions. In fact, exact computation of solutions of this type free discontinuity problems can be very rarely performed, with the exception of situations where some symmetries allow to reduce the problem to a one-dimensional problem. To approximate and compute solutions to variational problems (1.1) and (1.2), the most popular and successful approach is to use the theory of $\Gamma$-convergence [16]. This theory, introduced by De Giorgi and Franzoni in [18], is designed to approximate a variational problem by a sequence of different (usually, regularized) variational problems and ensures convergence of extremal values to extremal values and of minimizers to minimizers.

Several types of approximate variational problems for (1.1) and (1.2) have been studied extensively in the literature. At least three classes of $\Gamma$-convergent approximations to the functional (1.2) were proposed and analyzed in the literature. The first class is based on introducing higher-order singular perturbation terms ( $c f . ~[9]$ and the references therein); the second class approximates the functional (1.2) by non-local functionals, i.e., density functions are non-local ( $c f$. [10]). The idea of the third class of approximations, which was proposed by De Giorgi and developed by Gobbino (cf. [26] and reference therein), is to average the difference quotients among all possible directions in the functional. One such example is

$$
J_{\varepsilon}(u)=\frac{1}{\varepsilon} \int_{\Omega} \arctan \left(\frac{|u(x+\varepsilon)-u(x)|^{2}}{\varepsilon}\right) \mathrm{d} x+\frac{1}{2} \int_{\Omega} \gamma|u-g|^{2} \mathrm{~d} x .
$$

Following an earlier idea of Blake and Zissermann [7], Chambolle $[13,14]$ proposed a discrete finite-difference approximation and showed its $\Gamma$-convergence to the Mumford-Shah functional (1.1) in the one-dimensional case and to an anisotropic version of (1.1) in the two-dimensional case. Probably, the best known and commonly used approximation to the Mumford-Shah functional (1.1) is the following elliptic approximation due to Ambrosio and Tortorelli $[2,3]$ :

$$
A T_{\varepsilon}(u, \varphi)=\frac{\alpha}{2} \int_{\Omega}\left(k_{\varepsilon}+\varphi^{2}\right)|\nabla u|^{2} \mathrm{~d} x+\beta \int_{\Omega}\left[\varepsilon|\nabla \varphi|^{2}+\frac{1}{4 \varepsilon} f(\varphi)\right] \mathrm{d} x+\frac{1}{2} \int_{\Omega} \gamma|u-g|^{2} \mathrm{~d} x,
$$


for $f(\varphi)=(1-\varphi)^{2}$, and $0<k_{\varepsilon}=o(\varepsilon)$. The $\Gamma$-convergence of $A T_{\varepsilon}(u, \varphi)$ to $E(u, K)$ was established in $[2,3]$. The motivation for this approximation came from the Modica-Mortola theorem $[29,30]$ which enables the variational approximation of $P(E, \Omega)$, the perimeter of $E$ in $\Omega$, by the quadratic and elliptic Cahn-Hilliard functional [12]. A key idea of Ambrosio and Tortorelli's approximation is to replace the original "double well" potential $p(z)=z^{2}(1-z)^{2}$ in the Cahn-Hilliard functional by the quadratic "single well" potential $f(z)=(1-z)^{2}$ in order to approximate $\mathcal{H}^{N-1}(K)$ in (1.1). The function $\varphi$ in (1.4) can be regarded as a "phase function" so $K$ is roughly indicated by the set $\{\varphi \approx 0\}$, whereas its complement in $\Omega$ corresponds to $\{\varphi \approx 1\}$. Borrowing terminologies from phase transition in materials science, $A T_{\varepsilon}(u, \varphi)$ can be regarded as a phase field (diffuse interface) model to the Mumford-Shah (sharp interface) model $E(u, K)$.

Finite element approximations to the Mumford-Shah functional (1.1) based on the functional $A T_{\varepsilon}(u, \varphi)$ were first carried out by Bellettini and Coscia in [6]. It was shown that $A T_{\varepsilon}: V^{h}(\Omega) \times V^{h}(\Omega,[0,1]) \rightarrow \overline{\mathbf{R}}$ $\Gamma$-converges to $E: H^{1}(\Omega) \times L^{\infty}(\Omega) \rightarrow \overline{\mathbf{R}}$ under the condition that the mesh size $h=o\left(k_{\varepsilon}\right)$, provided that $S_{u}$ is piecewise smooth. Here $V^{h}(\Omega)$ denotes the continuous piecewise linear finite element space (cf. Sect. 3), and $V^{h}(\Omega,[0,1])=\left\{v_{h} \in V^{h}(\Omega) ; 0 \leq v_{h}(x) \leq 1 \forall x \in \Omega\right\}$. Later, Bourdin [8] extended the result of [6] to general $S_{u}$ by using a result of Dibos and Séré [19] for approximating the set $S_{u}$ by piecewise smooth hypersurfaces. Moreover, he showed that restricting functions $\varphi_{h} \in V^{h}(\Omega)$ to values in $[0,1]$ is not necessary since this constraint is already implicitly satisfied.

The main goal of this paper is to analyze the following gradient flow of the functional $A T_{\varepsilon}$ :

$$
\begin{aligned}
u_{t}-\alpha \operatorname{div}\left(\left(k_{\varepsilon}+\varphi^{2}\right) \nabla u\right)+\gamma(u-g)=0 & \text { in } \Omega_{T}:=(0, T) \times \Omega, \\
\varphi_{t}-2 \beta \varepsilon \Delta \varphi+\alpha|\nabla u|^{2} \varphi+\frac{\beta}{2 \varepsilon}(\varphi-1)=0 & \text { in } \Omega_{T}, \\
\frac{\partial u}{\partial n}=\frac{\partial \varphi}{\partial n}=0 & \text { on } \partial \Omega_{T}:=(0, T) \times \partial \Omega, \\
u(\cdot, 0)=u_{0}(\cdot), \quad \varphi(\cdot, 0)=\varphi_{0}(\cdot) & \text { in } \Omega,
\end{aligned}
$$

and its fully discrete finite element approximations. The primary motivation for considering the above gradient flow is to study the steepest descent method for minimizing the functional (1.4), which is often used in practice. In addition, a good understanding of the above gradient flow paves the way for us to analyze the gradient flow of the Mumford-Shah functional [23], in particular, in high dimensions. Numerical results of [21,28] have indicated various interesting stability and instability properties of the solutions of the gradient flow (1.5)-(1.8), for certain choices of parameters $\alpha, \beta, \gamma, \varepsilon, k_{\varepsilon}>0$. Another goal of this work is to understand these observations qualitatively by studying regularity and stability of the solutions of the above initial-boundary value problem, as well as error estimates of their finite element approximations (cf. [22]).

We note that in the one-dimensional case, the gradient flow for the Mumford-Shah functional was studied by Gobbino in [26], based on the nonlocal approximate functional (1.3). He established $\Gamma$-convergence of $J_{\varepsilon}(\cdot)$ to $\tilde{E}(\cdot)$ with respect to $L^{2}$-topology, derived the gradient flow for $\tilde{E}(\cdot)$ by setting $\varepsilon \rightarrow 0$, and proved that solutions to the limiting problem solve local heat equations, separated by an invariant set of jumps, i.e., $S(u(t)) \subseteq S\left(u_{0}\right)$, for all $t \geq 0$.

We also note that a slightly modified Mumford-Shah model has recently been proposed by Esedoglu and Shen [21] as an image inpainting model, and Ambrosio and Tortorelli's elliptic approximation was also used as a vehicle for numerical simulations. The proposed image inpainting model has exactly the same form as the Mumford-Shah model (1.1), and the proposed elliptic approximation has exactly the same form as the Ambrosio and Tortorelli's approximation. The only difference is that the parameter $\gamma$ in (1.1) and (1.4) now stands for the indicator function $\gamma_{D}(x)$ of $\Omega \backslash D$, where $D$ denotes the inpainted region of an image. We like to remark that, as a by-product, the results of the present paper also apply to this image inpainting model.

The paper is organized as follows. Section 2 devotes to analyzing the initial-boundary value problem (1.5)(1.8). We first establish existence of weak solutions for $u_{0}, g$ in $L^{2}(\Omega)$ and $\varphi_{0} \in L^{\infty}(\Omega)$, then prove that the system has a unique global in time strong solution, which has at most finite number of point singularities 
in the space-time, when the initial data $\left(u_{0}, \varphi_{0}\right) \in H^{1}(\Omega) \times H^{1}(\Omega) \cap L^{\infty}(\Omega)$. Our proof is based on a local energy idea due to Struwe [35]. A priori solution estimates are established in various norms, especially, by tracing their precise dependence on data $\left(u_{0}, \varphi_{0}\right)$ and $g$, as well as on parameters $\varepsilon, \alpha, \beta$ and $\gamma$. These results play a crucial role for understanding stability properties of the flow and for establishing error estimates for finite element approximations in the next section. Based on the analytical results of Section 2, Section 3 studies qualitatively finite element approximations of the gradient flow (1.5)-(1.8). We formulate and analyze a family of fully discrete finite element approximations using implicit Euler discretization in time, and continuous, piecewise linear finite element discretization in space. Optimal and suboptimal order error bounds, which show dependence on $\frac{1}{\varepsilon}$ and $\frac{1}{k_{\varepsilon}}$ only in low polynomial order, are derived for the proposed fully discrete schemes under the mesh relation $k=o\left(h^{\frac{1}{2}}\right)$. It is shown that semi-implicit treatment of the nonlinear term in (1.5) or in (1.6) results in schemes which satisfy a discrete energy law, while the same discrete energy law may not hold for the fully implicit scheme. On the other hand, as expected, the fully implicit scheme produces smaller errors than its semi-implicit counterparts do, although they have same asymptotic rate of convergence. Section 4 gathers numerical experiments derived from the introduced semi-discretization in Section 3 to study the gradient flow for different initial data.

\section{Analysis of the initial-Boundary value problem (1.5)-(1.8)}

The first goal of this section is to establish existence, uniqueness, stability and regularity properties for the gradient flow (1.5)-(1.8). The second goal is to derive a priori estimates, by tracing dependence on the parameters $\varepsilon, k_{\varepsilon}, \alpha, \beta$ and $\gamma\left(\right.$ or $\gamma_{D}$ ). The results of this section will serve as the theoretical foundation for analyzing space-time discretizations of (1.5)-(1.8) in the next section, they will also play a crucial role for studying the gradient flow of the Mumford-Shah functional in [23].

Standard function space and norm notation are used in this paper, we refer to $[1,15,27,34]$ for their precise definitions. Throughout this paper, unless stated otherwise, $C$ will be used to denote a generic positive constant, which is independent of the parameters $\varepsilon, k_{\varepsilon}, \alpha, \beta$ and $\gamma$, as well as data $u_{0}, \varphi_{0}, g$, and solution $(u, \varphi)$.

\subsection{Existence of weak solutions}

We begin this subsection with the definition of weak solutions to (1.5)-(1.8).

Definition 2.1. Let $\Omega \subset \mathbf{R}^{N}(N=2,3)$ be a domain with Lipschitz boundary $\partial \Omega$. For given data $\left(u_{0}, \varphi_{0}\right) \in$ $\left[L^{2}(\Omega)\right]^{2}$ with $0 \leq \varphi_{0} \leq 1$ a.e. in $\Omega$, and $g \in L^{2}\left((0, T) ; L^{2}(\Omega)\right)$, a pair of functions $(u, \varphi)$ is said to be a weak solution to (1.5)-(1.8) if $(u, \varphi) \in\left[L^{\infty}\left((0, T) ; L^{2}(\Omega)\right) \cap L^{2}\left((0, T) ; H^{1}(\Omega)\right)\right]^{2}$ satisfies (1.5)-(1.8) in distribution sense, and $0 \leq \varphi \leq 1$ a.e. in $\Omega_{T}$.

Remark 2.1. It is not hard to check that a weak solution $(u, \varphi)$ also satisfies $\left(u_{t}, \varphi_{t}\right) \in\left[L^{2}\left((0, T) ; H^{-2}(\Omega)\right)\right]^{2}$. An application of Aubin-Lions embedding lemma $[27,34]$ implies that $(u, \varphi) \in\left[C^{0}\left((0, T) ; L^{2}(\Omega)\right)\right]^{2}$, hence, two equations in (1.8) are well-defined.

The existence of a weak solution is given by the following theorem.

Theorem 2.1. Given $\left(u_{0}, \varphi_{0}\right) \in\left[L^{2}(\Omega)\right]^{2}$ with $0 \leq \varphi_{0} \leq 1$ a.e. in $\Omega$, and $g \in L^{2}\left((0, T) ; L^{2}(\Omega)\right)$, let $\tau(t)$ be any positive $C^{1}$ function on $[0, T]$ such that $\tau(0)=0$. Then, (1.5)-(1.8) possesses a weak solution $(u, \varphi)$ which satisfies for $s \in[0, T]$

$$
\begin{aligned}
& \|u(s)\|_{L^{2}}^{2}+\int_{0}^{s}\left[2 \alpha\left(k_{\varepsilon}\|\nabla u\|_{L^{2}}^{2}+\|\varphi \nabla u\|_{L^{2}}^{2}\right)+\gamma\|u\|_{L^{2}}^{2}\right] \mathrm{d} t \leq \mathcal{B}_{0} \\
& \|\varphi(s)\|_{L^{2}}^{2}+\int_{0}^{s}\left[4 \beta \varepsilon\|\nabla \varphi\|_{L^{2}}^{2}+2 \alpha\|\varphi \nabla u\|_{L^{2}}^{2}+\frac{\beta}{2 \varepsilon}\|f(\varphi)\|_{L^{1}}\right] \mathrm{d} t \leq \mathcal{B}_{1}, \\
& \tau(s) A T_{\varepsilon}(u(s), \varphi(s))+\int_{0}^{s} \tau(t)\left[\left\|u_{t}\right\|_{L^{2}}^{2}+\left\|\varphi_{t}\right\|_{L^{2}}^{2}\right] \mathrm{d} t=\int_{0}^{s} \tau^{\prime}(t) A T_{\varepsilon}(u, \varphi) \mathrm{d} t,
\end{aligned}
$$


where

$$
\mathcal{B}_{0}:=\left\|u_{0}\right\|_{L^{2}}^{2}+\|\sqrt{\gamma} g(t)\|_{L^{2}\left(L^{2}\right)}^{2}, \quad \mathcal{B}_{1}:=\left\|\varphi_{0}\right\|_{L^{2}}^{2}+\beta|\Omega| \varepsilon^{-1} .
$$

Moreover, if $u_{0} \in L^{\infty}(\Omega)$ and $g \in L^{\infty}\left(\Omega_{T}\right)$, then $u$ satisfies the following weak maximum principle,

$$
\min \left\{\min _{\Omega_{T}} g, \min _{\Omega} u_{0}\right\} \leq u(x, t) \leq \max \left\{\max _{\Omega_{T}} g, \max _{\Omega} u_{0}\right\} \quad \text { a.e. }(x, t) \in \Omega_{T} .
$$

Proof. Since solvability follows easily from applying the standard energy method [27] provided that a priori estimates (2.1)-(2.3) and $0 \leq \varphi \leq 1$ can be verified, we only give a proof for these estimates in the following.

Since $\varphi$ and $\psi:=\varphi-1$ satisfy, respectively,

$$
\begin{aligned}
& \varphi_{t}-2 \beta \varepsilon \Delta \varphi+\left[\alpha|\nabla u|^{2}+\frac{\beta}{2 \varepsilon}\right] \varphi \geq 0, \quad \varphi(0)=\varphi_{0} \geq 0 \\
& \psi_{t}-2 \beta \varepsilon \Delta \psi+\left[\alpha|\nabla u|^{2}+\frac{\beta}{2 \varepsilon}\right] \psi \leq 0, \quad \psi(0)=\varphi_{0}-1 \leq 0
\end{aligned}
$$

testing the first equation by $\varphi^{-}:=\max \{-\varphi, 0\}$ and the second by $\psi^{+}:=\max \{\psi, 0\}$, and using the fact that $\varphi(0)^{-}=0$ and $\psi(0)^{+}=0$ immediately yields $0 \leq \varphi(t, x) \leq 1$, a.e. $(t, x) \in \Omega_{T}$.

Next, (2.1) and (2.2) follows directly from testing (1.5) by $u$ and (1.6) by $\varphi$, respectively.

To show (2.3), testing (1.5) by $\tau(t) u_{t}$ and (1.6) by $\tau(t) \varphi_{t}$, and adding the resulted equations we get

$$
\frac{\mathrm{d}}{\mathrm{d} t}\left[\tau(t) A T_{\varepsilon}(u(t), \varphi(t))\right]+\tau(t)\left[\left\|u_{t}(t)\right\|_{L^{2}}^{2}+\left\|\varphi_{t}(t)\right\|_{L^{2}}^{2}\right]=\tau^{\prime}(t) A T_{\varepsilon}(u(t), \varphi(t)) .
$$

Then (2.3) follows from integrating (2.5) in $t$ from 0 to $s$ and using the assumption $\tau(0)=0$.

Finally, it remains to verify (2.4). Define

$$
\kappa_{1}:=\min \left\{\min _{\Omega_{T}} g, \min _{\Omega} u_{0}\right\}, \quad \kappa_{2}:=\max \left\{\max _{\Omega_{T}} g, \max _{\Omega} u_{0}\right\} .
$$

The assertion immediately follows from testing $(1.5)$ by $\left(u-\kappa_{1}\right)^{-}$and $\left(u-\kappa_{2}\right)^{+}$, respectively. The proof is complete.

Remark 2.2. (a). Note that $\mathcal{B}_{0}$ is independent of $\varepsilon$, but $\mathcal{B}_{0}$ grows linearly in $\varepsilon^{-1}$. This is expectable since $\varphi$ should have large gradient (corresponding to small $\varepsilon$ ) near the edges of an image. Since (2.2) implies that $\|\nabla \varphi\|_{L^{2}\left(\Omega_{T}\right)}=O\left(\varepsilon^{-1}\right)$, we conclude that the width of smeared edges of Ambrosio-Tortorelli's approximation model (1.4) is not bigger than $O(\varepsilon)$ order.

(b). Since $u_{0} \in L^{\infty}(\Omega)$ and $g \in L^{\infty}\left(\Omega_{T}\right)$ are satisfied for all image applications, practically, (2.4) holds in general.

\subsection{Regularity and uniqueness properties of weak solutions}

In this section we address regularity and uniqueness properties of weak solutions to (1.5)-(1.8) with more regular datum functions. Special attention will be given on deriving a priori estimates with explicit dependence on the parameters $\varepsilon, k_{\varepsilon}, \alpha, \beta, \gamma$, and the data $\left(u_{0}, \varphi_{0}\right)$ and $g$. Such a priori estimates will be useful for finite element error analysis in the next section. It turns out that estimates in $L^{2}\left(\Omega_{T}\right)$ of all first order derivatives of the solutions are easy to get due to the fact that there is an underlying energy law for every gradient flow. However, it is far from straightforward to derive a priori estimates for the second order derivatives of the solution due to the strong nonlinearity. To overcome the difficulty, we use an idea of Struwe [35] to examine the solution's behavior based on a local energy law, and show that the system has a unique global in time strong solution which is (strongly) differentiable in space-time, away from at most finitely many points $\left\{\left(\bar{x}_{\ell}, \bar{t}_{\ell}\right)\right\}_{\ell=1}^{K}$ in $\Omega_{T}$. 
We start with the energy law for the gradient flow (1.5)-(1.8).

Theorem 2.2. In addition to assumptions of Theorem 2.1, suppose that $\left(u_{0}, \varphi_{0}\right) \in\left[H^{1}(\Omega)\right]^{2}$, and $g \in$ $L^{2}(\Omega)$ is independent of $t$. Then, weak solutions $(u, \varphi)$ to $(1.5)-(1.8)$ satisfy $(u, \varphi) \in\left[L^{\infty}\left((0, T) ; H^{1}(\Omega)\right) \cap\right.$ $\left.H^{1}\left((0, T) ; L^{2}(\Omega)\right)\right]^{2}$ and the energy law

$$
\underset{t \in[0, T]}{\operatorname{ess} \sup } A T_{\varepsilon}(u(t), \varphi(t))+\int_{0}^{T}\left[\left\|\varphi_{t}\right\|_{L^{2}}^{2}+\left\|u_{t}\right\|_{L^{2}}^{2}\right] \mathrm{d} t=A T_{\varepsilon}\left(u_{0}, \varphi_{0}\right) .
$$

Proof. It suffices to prove (2.6), which follows in the same way as for (2.3) (cf. (2.5)) with $\tau(t) \equiv 1$. Since $A T_{\varepsilon}\left(u_{0}, \varphi_{0}\right)<\infty,(2.6)$ follows from integrating (2.5) in $t$ from 0 to $s \in[0, T]$. The proof is complete.

Definition 2.2. A weak solution $(u, \varphi)$ is called a quasi-strong solution if $(u, \varphi) \in\left[H^{1}\left((0, T) ; L^{2}(\Omega)\right) \cap\right.$ $\left.L^{\infty}\left((0, T) ; H^{1}(\Omega)\right)\right]^{2}$.

For $x_{0} \in \Omega$, let $B_{R}\left(x_{0}\right)$ denote the ball of radius $R(>0)$ centered at $x_{0}$. Define the local energy over $B_{R}\left(x_{0}\right)$ as

$$
\begin{aligned}
A T_{\varepsilon}\left((u(t), \varphi(t)) ; B_{R}\left(x_{0}\right)\right)=\frac{\alpha}{2} \int_{B_{R}\left(x_{0}\right)}\left(k_{\varepsilon}\right. & \left.+\varphi^{2}\right)|\nabla u|^{2} \mathrm{~d} x \\
& +\beta \int_{B_{R}\left(x_{0}\right)}\left[\varepsilon|\nabla \varphi|^{2}+\frac{1}{4 \varepsilon} f(\varphi)\right] \mathrm{d} x+\frac{1}{2} \int_{B_{R}\left(x_{0}\right)} \gamma|u-g|^{2} \mathrm{~d} x .
\end{aligned}
$$

Next, we show that a local version of (2.6), referred as the local energy law, also holds for the gradient flow (1.5)-(1.8).

Lemma 2.1. Let $\left(u_{0}, \varphi_{0}\right) \in\left[H^{1}(\Omega)\right]^{2}$. For $x_{0} \in \Omega$, let $B_{2 R_{0}}\left(x_{0}\right)$ be the largest ball contained in $\Omega$ with the center at $x_{0}$. Then, for all $0<R \leq R_{0}$, there holds

$$
A T_{\varepsilon}\left((u(T), \varphi(T)) ; B_{R}\left(x_{0}\right)\right) \leq A T_{\varepsilon}\left(\left(u_{0}, \varphi_{0}\right) ; B_{2 R}\left(x_{0}\right)\right)+\frac{\mathcal{B}_{2} T}{R^{2}} A T_{\varepsilon}\left(u_{0}, \varphi_{0}\right) \quad \forall T \geq 0,
$$

where $\mathcal{B}_{2}:=16\left[\alpha\left(1+k_{\varepsilon}\right)+\beta \varepsilon\right]$.

Proof. Let $\phi$ be a cutoff function satisfying $\phi \in C_{0}^{\infty}\left(B_{2 R}\left(x_{0}\right)\right), 0 \leq \phi \leq 1$ in $\Omega, \phi \equiv 1$ in $B_{R}\left(x_{0}\right)$, and $|\nabla \phi| \leq \frac{2}{R}$. Testing (1.5)-(1.6) with $\left(\phi^{2} u_{t}, \phi^{2} \varphi_{t}\right)$ and applying Young's inequality lead to

$$
\begin{gathered}
\int_{\Omega}\left[\left|u_{t}\right|^{2}+\left|\varphi_{t}\right|^{2}\right] \phi^{2} \mathrm{~d} x+\frac{\mathrm{d}}{\mathrm{d} t}\left[\frac{k_{\varepsilon} \alpha}{2} \int_{\Omega}|\nabla u|^{2} \phi^{2} \mathrm{~d} x+\beta \varepsilon \int_{\Omega}|\nabla \varphi|^{2} \phi^{2} \mathrm{~d} x\right. \\
\left.+\frac{\alpha}{2} \int_{\Omega}|\nabla u|^{2} \varphi^{2} \phi^{2} \mathrm{~d} x+\frac{1}{2} \int_{\Omega} \gamma|u-g|^{2} \phi^{2} \mathrm{~d} x+\frac{\beta}{4 \varepsilon} \int_{\Omega}|\varphi-1|^{2} \phi^{2} \mathrm{~d} x\right] \\
\leq 2 k_{\varepsilon} \alpha\left|\left(\nabla u, \phi \nabla \phi u_{t}\right)\right|+4 \beta \varepsilon\left|\left(\nabla \varphi, \phi \nabla \phi \varphi_{t}\right)\right|+2 \alpha\left|\left(\varphi^{2} \nabla u, \phi \nabla \phi u_{t}\right)\right| \\
\leq 2 k_{\varepsilon}^{2} \alpha^{2} \int_{\Omega}|\nabla u|^{2}|\nabla \varphi|^{2} \mathrm{~d} x+4 \beta^{2} \varepsilon^{2} \int_{\Omega}|\nabla \varphi|^{2}|\nabla \phi|^{2} \mathrm{~d} x+2 \alpha^{2} \int_{\Omega} \varphi^{4}|\nabla u|^{2}|\nabla \phi|^{2} \mathrm{~d} x+\int_{\Omega}\left[\left|u_{t}\right|^{2}+\left|\varphi_{t}\right|^{2}\right] \phi^{2} \mathrm{~d} x \\
\leq \frac{16}{R^{2}}\left[k_{\varepsilon} \alpha+\alpha+\beta \varepsilon\right] A T_{\varepsilon}\left(u_{0}, \varphi_{0}\right)+\int_{\Omega}\left[\left|u_{t}\right|^{2}+\left|\varphi_{t}\right|^{2}\right] \phi^{2} \mathrm{~d} x .
\end{gathered}
$$

Integration over time then implies the assertion. 
To proceed, we need some notation. Suppose $\left(u_{0}, \varphi_{0}\right) \in\left[H^{1}(\Omega)\right]^{2}$, for any given $\varepsilon_{1}>0$, let $R_{1}>0$ be the maximal number such that

$$
\sup _{x_{0} \in \Omega} A T_{\varepsilon}\left(\left(u_{0}, \varphi_{0}\right) ; B_{2 R_{1}}\left(x_{0}\right)\right)<\varepsilon_{1}
$$

and let $T_{1}>0$ be a number such that any weak solution $(u, \varphi)$ of $(1.5)-(1.8)$ taking the initial value $\left(u_{0}, \varphi_{0}\right)$ satisfies

$$
\sup _{x_{0} \in \Omega} A T_{\varepsilon}\left((u(t), \varphi(t)) ; B_{R_{1}}\left(x_{0}\right)\right)<2 \varepsilon_{1} .
$$

Note that, in view of the local energy law of Lemma 2.1, we may let $T_{1}=\frac{\varepsilon_{1} R_{1}^{2}}{\mathcal{B}_{2} A T_{\varepsilon}\left(u_{0}, \varphi_{0}\right)}$.

Theorem 2.3. Let $N=2$, suppose that $A T_{\varepsilon}\left(u_{0}, \varepsilon_{0}\right)<\infty$. For sufficiently small $\varepsilon_{1}=O\left(\varepsilon k_{\varepsilon}^{2}\right)$, choose $T_{1}$ as above, (1.5)-(1.8) has a unique strong solution $(u, \varphi) \in\left[L^{\infty}\left(\left(0, T_{1}\right) ; H^{1}(\Omega)\right) \cap L^{2}\left(\left(0, T_{1}\right) ; H^{2}(\Omega)\right)\right]^{2}$ which satisfies

$$
\begin{aligned}
& \max _{\left[0, T_{1}\right)}\left(\|\nabla u\|_{L^{2}}^{2}+\|\nabla \varphi\|_{L^{2}}^{2}\right)+\int_{0}^{T_{1}}\left(\alpha\left\|\sqrt{k_{\varepsilon}^{2}+\varphi^{2}} \Delta u\right\|_{L^{2}}^{2}+\beta \varepsilon\|\Delta \varphi\|_{L^{2}}^{2}\right) \mathrm{d} s \\
& \quad \leq C_{0}:=\frac{C \varepsilon k_{\varepsilon}}{\mathcal{B}_{2}}\left[\frac{R_{1}^{2}}{A T_{\varepsilon}\left(u_{0}, \varphi_{0}\right)}\|\gamma g\|_{L^{2}}^{2}+k_{\varepsilon}\right] .
\end{aligned}
$$

Proof. It suffices to derive the desired a priori error estimate and prove the uniqueness. We divide the proof into three steps.

Step 1. Existence: a first a priori bound. Testing equations (1.5)-(1.6) with $(-\Delta u,-\Delta \varphi)$ gives

$$
\begin{aligned}
\frac{1}{2} \frac{\mathrm{d}}{\mathrm{d} t}\left(\|\nabla u\|_{L^{2}}^{2}+\|\nabla \varphi\|_{L^{2}}^{2}\right)+\frac{\alpha}{2}\left\|\sqrt{k_{\varepsilon}+\varphi^{2}} \Delta u\right\|_{L^{2}}^{2}+2 \beta \varepsilon\|\Delta \varphi\|_{L^{2}}^{2}+\frac{\beta}{2 \varepsilon}\|\nabla \varphi\|_{L^{2}}^{2}+\|\sqrt{\gamma} \nabla u\|_{L^{2}}^{2} \\
\leq \frac{1}{2 \alpha k_{\varepsilon}}\|\gamma g\|_{L^{2}}^{2}+2 \alpha|(\varphi \nabla \varphi, \nabla u \Delta u)|+\alpha\left|\left(|\nabla u|^{2} \varphi, \Delta \varphi\right)\right| .
\end{aligned}
$$

The last two terms can be bounded as follows

$$
\begin{aligned}
2 \alpha|(\varphi \nabla \varphi, \nabla u \Delta u)|+\alpha\left|\left(|\nabla u|^{2} \varphi, \Delta \varphi\right)\right| \leq \frac{\alpha}{4}\|\varphi \Delta u\|_{L^{2}}^{2}+\beta \varepsilon\|\Delta \varphi\|_{L^{2}}^{2} & \\
& +2 \alpha\|\nabla \varphi\|_{L^{4}}^{4}+\left[2 \alpha+\frac{\alpha^{2}}{4 \beta \varepsilon}\right]\|\nabla u\|_{L^{4}}^{4}
\end{aligned}
$$

Step 2. Existence: control of $(\nabla u, \nabla \varphi)$. Let $\left\{\phi_{i}\right\}_{i \geq 1}$ be a smooth partition of unity subordinate to a cover of $\Omega$ by balls $\left\{B_{R_{1}}\left(x_{i}\right)\right\}_{i \geq 1}$ with finite overlaps satisfying $0 \leq \phi_{i} \leq 1,\left|\nabla \phi_{i}\right| \leq \frac{2}{R_{1}}$, and $\sum_{i \geq 1} \phi_{i}^{2}=1$. Then, interpolating $L^{4}$ norm by $L^{2}$ and $H^{1}$ norms and using (2.9) we have

$$
\begin{aligned}
\|\nabla \varphi(t)\|_{L^{4}}^{4} & =\sum_{i \geq 1} \int_{\Omega}|\nabla \varphi(t)|^{4} \phi_{i}^{2} \mathrm{~d} x \\
& \leq C \sum_{i \geq 1}\|\nabla \varphi\|_{L^{2}\left(B_{R_{1}}\left(x_{i}\right)\right)}^{2}\left[\left\|\nabla^{2} \varphi\right\|_{L^{2}\left(B_{R_{1}}\left(x_{i}\right)\right)}^{2}+\left\||\nabla \varphi|\left|\nabla \phi_{i}\right|\right\|_{L^{2}\left(B_{R_{1}}\left(x_{i}\right)\right)}^{2}\right] \\
& \leq \frac{C}{\beta \varepsilon} \sup _{i} A T_{\varepsilon}\left((u(t), \varphi(t)) ; B_{R_{1}}\left(x_{i}\right)\right)\left[\left\|\nabla^{2} \varphi(t)\right\|_{L^{2}}^{2}+\frac{1}{\beta \varepsilon R_{1}^{2}} A T_{\varepsilon}\left(u_{0}, \varphi_{0}\right)\right] \\
& \leq \frac{C \varepsilon_{1}}{\beta \varepsilon}\left[\|\Delta \varphi(t)\|_{L^{2}}^{2}+\frac{1}{\beta \varepsilon R_{1}^{2}} A T_{\varepsilon}\left(u_{0}, \varphi_{0}\right)\right] \quad \forall t \in\left[0, T_{1}\right) .
\end{aligned}
$$


Here we have used the Calderón-Zygmund inequality to get the last inequality. Similarly, we have

$$
\|\nabla u(t)\|_{L^{4}}^{4} \leq \frac{C \varepsilon_{1}}{k_{\varepsilon}}\left[\|\Delta u(t)\|_{L^{2}}^{2}+\frac{1}{k_{\varepsilon} R_{1}^{2}} A T_{\varepsilon}\left(u_{0}, \varphi_{0}\right)\right] \quad \forall t \in\left[0, T_{1}\right) .
$$

We recall that $T_{1}$ depends on $\varepsilon_{1}$ linearly in the form $T_{1}=\frac{\varepsilon_{1} R_{1}^{2}}{\mathcal{B}_{2} A T_{\varepsilon}\left(u_{0}, \varphi_{0}\right)}$.

Substituting (2.11)-(2.13) into (2.10) yields

$$
\begin{aligned}
\frac{1}{2} \frac{\mathrm{d}}{\mathrm{d} t}\left(\|\nabla u\|^{2}+\|\nabla \varphi\|^{2}\right)+ & \frac{\alpha}{4}\left\|\sqrt{k_{\varepsilon}+\varphi^{2}} \Delta u\right\|_{L^{2}}^{2}+\beta \varepsilon\|\Delta \varphi\|_{L^{2}}^{2}+\|\sqrt{\gamma} \nabla u\|_{L^{2}}^{2}+\frac{\beta}{2 \varepsilon}\|\nabla \varphi\|_{L^{2}}^{2} \\
\leq \frac{1}{2 \alpha k_{\varepsilon}}\|\gamma g\|_{L^{2}}^{2}+\frac{2 \alpha \varepsilon_{1} C}{\beta \varepsilon}\left[\|\Delta \varphi(t)\|_{L^{2}}^{2}+\frac{1}{\beta \varepsilon R_{1}^{2}} A T_{\varepsilon}\left(u_{0}, \varphi_{0}\right)\right] & +\left(\frac{\alpha(8 \beta \varepsilon+\alpha) \varepsilon_{1} C}{4 \beta \varepsilon k_{\varepsilon}}\right)\left[\|\Delta u(t)\|_{L^{2}}^{2}+\frac{1}{k_{\varepsilon} R_{1}^{2}} A T_{\varepsilon}\left(u_{0}, \varphi_{0}\right)\right] .
\end{aligned}
$$

Then, the desired estimate follows from integrating in $t$ from 0 to $T_{1}$ after choosing $\varepsilon_{1}=O\left(\varepsilon k_{\varepsilon}^{2}\right)$.

Step 3. Uniqueness: We now show that the space $\left[L^{\infty}\left((0, T) ; H^{1}(\Omega)\right) \cap L^{2}\left((0, T) ; H^{2}(\Omega)\right)\right]^{2}$ is a uniqueness class. Suppose $\left(u_{i}, \varphi_{i}\right)$ for $i=1,2$ are two strong solutions corresponding to the same datum functions $\left(u_{0}, \varphi_{0}\right)$, and $g$. Let $e=u_{1}-u_{2}$ and $\eta=\varphi_{1}-\varphi_{2}$, subtracting equations satisfied by $\left(u_{i}, \varphi_{i}\right)$ leads to the following "error" equations which hold in distributional sense,

$$
\begin{aligned}
e_{t}-\alpha \operatorname{div}\left(\left(k_{\varepsilon}+\varphi_{1}^{2}\right) \nabla e+\eta\left(\varphi_{1}+\varphi_{2}\right) \nabla u_{2}\right)+\gamma e=0 & \text { in } \Omega_{T}, \\
\eta_{t}-2 \beta \varepsilon \Delta \eta+\left(\alpha\left|\nabla u_{1}\right|^{2}+\frac{\beta}{2 \varepsilon}\right) \eta+\alpha\left(\left|\nabla u_{1}\right|^{2}-\left|\nabla u_{2}\right|^{2}\right) \varphi_{2}=0 & \text { in } \Omega_{T}, \\
\frac{\partial e}{\partial n}=\frac{\partial \eta}{\partial n}=0 & \text { on } \partial \Omega_{T}, \\
e(\cdot, 0)=\eta(\cdot, 0)=0 & \text { in } \Omega .
\end{aligned}
$$

Testing (2.14) by $e$ and (2.15) by $\eta$ we obtain

$$
\begin{aligned}
& \frac{1}{2} \frac{\mathrm{d}}{\mathrm{d} t}\|e\|_{L^{2}}^{2}+\alpha k_{\varepsilon}\|\nabla e\|_{L^{2}}^{2}+\alpha\left\|\varphi_{1} \nabla e\right\|_{L^{2}}^{2}+\|\sqrt{\gamma} e\|_{L^{2}}^{2}=-\alpha\left(\left(\varphi_{1}+\varphi_{2}\right) \nabla u_{2} \eta, \nabla e\right) \\
& \frac{1}{2} \frac{\mathrm{d}}{\mathrm{d} t}\|\eta\|_{L^{2}}^{2}+2 \beta \varepsilon\|\nabla \eta\|_{L^{2}}^{2}+\alpha\left\|\nabla u_{1} \eta\right\|_{L^{2}}^{2}+\frac{\beta}{2 \varepsilon}\|\eta\|_{L^{2}}^{2}=-\alpha\left(\left[\left|\nabla u_{1}\right|^{2}-\left|\nabla u_{2}\right|^{2}\right] \varphi_{2}, \eta\right)
\end{aligned}
$$

Reversing the roles of $\left(u_{1}, \varphi_{1}\right)$ and $\left(u_{2}, \varphi_{2}\right)$ in the above derivation then gives

$$
\begin{gathered}
\frac{1}{2} \frac{\mathrm{d}}{\mathrm{d} t}\|e\|_{L^{2}}^{2}+\alpha k_{\varepsilon}\|\nabla e\|_{L^{2}}^{2}+\alpha\left\|\varphi_{2} \nabla e\right\|_{L^{2}}^{2}+\|\sqrt{\gamma} e\|_{L^{2}}^{2}=-\alpha\left(\left(\varphi_{1}+\varphi_{2}\right) \nabla u_{1} \eta, \nabla e\right) \\
\begin{array}{c}
\frac{1}{2} \frac{\mathrm{d}}{\mathrm{d} t}\|\eta\|_{L^{2}}^{2}+2 \beta \varepsilon\|\nabla \eta\|_{L^{2}}^{2}+\alpha\left\|\nabla u_{2} \eta\right\|_{L^{2}}^{2}+\frac{\beta}{2 \varepsilon}\|\eta\|_{L^{2}}^{2} \\
=-\alpha\left(\left[\left|\nabla u_{1}\right|^{2}-\left|\nabla u_{2}\right|^{2}\right] \varphi_{1}, \eta\right) .
\end{array}
\end{gathered}
$$


Now, adding (2.20) to (2.18), and (2.21) to (2.19), we finally get

$$
\begin{aligned}
\frac{\mathrm{d}}{\mathrm{d} t}\|e\|_{L^{2}}^{2}+2 \alpha k_{\varepsilon}\|\nabla e\|_{L^{2}}^{2}+\alpha\left[\left\|\varphi_{1} \nabla e\right\|_{L^{2}}^{2}+\left\|\varphi_{2} \nabla e\right\|_{L^{2}}^{2}\right]+2\|\sqrt{\gamma} e\|_{L^{2}}^{2} & =-\alpha\left(\left(\varphi_{1}+\varphi_{2}\right)\left(\nabla u_{1}+\nabla u_{2}\right) \eta, \nabla e\right), \\
\frac{\mathrm{d}}{\mathrm{d} t}\|\eta\|_{L^{2}}^{2}+4 \beta \varepsilon\|\nabla \eta\|_{L^{2}}^{2}+\alpha\left[\left\|\nabla u_{1} \eta\right\|_{L^{2}}^{2}+\left\|\nabla u_{2} \eta\right\|_{L^{2}}^{2}\right]+\frac{\beta}{\varepsilon}\|\eta\|_{L^{2}}^{2} & =-\alpha\left(\left[\left|\nabla u_{1}\right|^{2}-\left|\nabla u_{2}\right|^{2}\right]\left(\varphi_{1}+\varphi_{2}\right), \eta\right) \\
& =-\alpha\left(\left(\varphi_{1}+\varphi_{2}\right)\left(\nabla u_{1}+\nabla u_{2}\right) \eta, \nabla e\right) .
\end{aligned}
$$

We bound the term on the right hand sides of (2.23) and (2.24) as follows,

$$
\begin{aligned}
\left|2 \alpha\left(\left(\varphi_{1}+\varphi_{2}\right)\left(\nabla u_{1}+\nabla u_{2}\right) \eta, \nabla e\right)\right| & \leq 2 \alpha\left\|\varphi_{1}+\varphi_{2}\right\|_{L^{\infty}}\|\nabla e\|_{L^{2}}\left\|\nabla\left(u_{1}+u_{2}\right)\right\|_{L^{4}}\|\eta\|_{L^{4}} \\
& \leq \frac{8 \alpha}{k_{\varepsilon}}\left\|\nabla\left(u_{1}+u_{2}\right)\right\|_{L^{4}}^{2}\|\eta\|_{L^{4}}^{2}+\frac{\alpha k_{\varepsilon}}{2}\|\nabla e\|_{L^{2}}^{2} \\
& \leq \frac{8 C \alpha^{2}}{\beta \varepsilon k_{\varepsilon}^{2}}\left\|\nabla\left(u_{1}+u_{2}\right)\right\|_{L^{4}}^{4}\|\eta\|_{L^{2}}^{2}+2 \beta \varepsilon\|\nabla \eta\|_{L^{2}}^{2}+\frac{\alpha k_{\varepsilon}}{2}\|\nabla e\|_{L^{2}}^{2}
\end{aligned}
$$

Since $u_{i} \in L^{2}\left((0, T) ; H^{2}(\Omega)\right) \subset L^{4}\left((0, T) ; W^{1,4}(\Omega)\right)$, it follows from adding $(2.23)$ to $(2.24)$, using (2.25) and the Gronwall's inequality that

Hence, uniqueness follows.

$$
\|e(t)\|_{L^{2}}^{2}+\|\eta(t)\|_{L^{2}}^{2}=0 \quad \forall t \in\left[0, T^{*}\right]
$$

Remark 2.3. Step 3 above actually shows that $\left[L^{\infty}\left((0, T) ; H^{1}(\Omega)\right) \cap L^{4}\left((0, T) ; W^{1,4}(\Omega)\right)\right]^{2}$ is a uniqueness class in both cases $N=2$ and $N=3$.

The following lemma is now a consequence of Theorem 2.3.

Corollary 2.1. Let $N=2$, and $\varepsilon_{1}, T_{1}$ be same as in Theorem 2.3. Suppose $\left(u_{0}, \varphi_{0}\right) \in\left[H^{2}(\Omega)\right]^{2}$. Then, $(u, \varphi) \in\left[L^{\infty}\left(\left(0, T_{1}\right) ; H^{2}(\Omega)\right)\right]^{2}$, and

$$
\begin{aligned}
& \text { (i) } \underset{\left[0, T_{1}\right)}{\operatorname{ess} \sup }\left(\left\|u_{t}\right\|_{L^{2}}^{2}+\left\|\varphi_{t}\right\|_{L^{2}}^{2}\right)+\int_{0}^{T_{1}}\left[\alpha\left\|\sqrt{k_{\varepsilon}+\varphi^{2}} \nabla u_{t}\right\|_{L^{2}}^{2}+\alpha\|\varphi t \nabla u\|_{L^{2}}^{2}+\beta \varepsilon\left\|\nabla \varphi_{t}\right\|_{L^{2}}^{2}\right] \mathrm{d} s \\
& \leq C_{1}:=\exp \left(\frac{\alpha\left(\mathcal{B}_{2} C_{0}+\varepsilon_{1}\right) C}{\beta \mathcal{B}_{2}}\right)\left[\left\|u_{t}(0)\right\|_{L^{2}}^{2}+\left\|\varphi_{t}(0)\right\|_{L^{2}}^{2}\right] \text {. } \\
& \text { (ii) } \operatorname{ess~sup}_{\left[0, T_{1}\right)}\left(\alpha^{2} k_{\varepsilon}\left\|\sqrt{k_{\varepsilon}+\varphi^{2}} \Delta u\right\|_{L^{2}}^{2}+\beta^{2} \varepsilon^{2}\|\Delta \varphi\|_{L^{2}}^{2}\right) \leq C_{2}:=C_{1}+\frac{\alpha^{2} \varepsilon\left(1+k_{\varepsilon}\right) C}{R_{1}^{2}}, \\
& \text { (iii) } \int_{0}^{T_{1}}\left\|u_{t t}\right\|_{H^{-1}}^{2} \mathrm{~d} s \leq C_{3}:=2 C_{1}+2\|\gamma\|_{L^{\infty}}^{2} A T_{\varepsilon}\left(u_{0}, \varphi_{0}\right) \\
& \text { (iii) } \int_{0}^{T_{1}}\left\|\varphi_{t t}\right\|_{H^{-1}}^{2} \mathrm{~d} s \leq C_{4}:=C C_{1}\left[8 \beta\left(\varepsilon+\beta k_{\varepsilon}\right)+\sqrt{\frac{C_{2} A T_{\varepsilon}\left(u_{0}, \varphi_{0}\right)}{\alpha k_{\varepsilon}^{3}}}\right] \text {. }
\end{aligned}
$$

Proof. (i) Differentiating (1.5) and (1.6) in $t$ yields

$$
\begin{aligned}
u_{t t}-\alpha \operatorname{div}\left(\left(k_{\varepsilon}+\varphi^{2}\right) \nabla u_{t}+2 \varphi \varphi_{t} \nabla u\right)+\gamma u_{t}=0 & \text { in } \Omega_{T}, \\
\varphi_{t t}-2 \beta \varepsilon \Delta \varphi_{t}+2 \alpha\left\langle\nabla u, \nabla u_{t}\right\rangle \varphi+\alpha|\nabla u|^{2} \varphi_{t}+\frac{\beta}{2 \varepsilon} \varphi_{t}=0 & \text { in } \Omega_{T} .
\end{aligned}
$$


Testing (2.26) with $u_{t}$ and (2.27) with $\varphi_{t}$, and adding the resulting equations lead to

$$
\begin{aligned}
\frac{1}{2} \frac{\mathrm{d}}{\mathrm{d} t}\left(\left\|u_{t}\right\|_{L^{2}}^{2}+\left\|\varphi_{t}\right\|_{L^{2}}^{2}\right)+\alpha\left\|\sqrt{k_{\varepsilon}+\varphi^{2}} \nabla u_{t}\right\|_{L^{2}}^{2}+\alpha\left\|\varphi_{t} \nabla u\right\|_{L^{2}}^{2}+2 \beta \varepsilon\left\|\nabla \varphi_{t}\right\|_{L^{2}}^{2}+\frac{\beta}{2 \varepsilon}\left\|\varphi_{t}\right\|_{L^{2}}^{2}+\left\|\sqrt{\gamma} u_{t}\right\|_{L^{2}}^{2} \\
\leq \frac{C \alpha^{2}}{\beta \varepsilon}\|\nabla u\|_{L^{4}}^{4}\left\|\varphi_{t}\right\|_{L^{2}}^{2}+\beta \varepsilon\left\|\nabla \varphi_{t}\right\|_{L^{2}}^{2}+\frac{\alpha}{2}\left\|\varphi \nabla u_{t}\right\|_{L^{2}}^{2} .
\end{aligned}
$$

By (2.13), the first term on the right hand side can be bounded as

$$
\frac{C \alpha^{2}}{\beta \varepsilon}\|\nabla u\|_{L^{4}}^{4}\left\|\varphi_{t}\right\|_{L^{2}}^{2} \leq \frac{C \alpha^{2} \varepsilon_{1}}{\beta \varepsilon k_{\varepsilon}}\left[\|\Delta u(t)\|_{L^{2}}^{2}+\frac{1}{k_{\varepsilon} R_{1}^{2}} A T_{\varepsilon}\left(u_{0}, \varphi_{0}\right)\right]\left\|\varphi_{t}\right\|_{L^{2}}^{2} .
$$

The regularity of the initial data ensures the existence of $\lim _{s \rightarrow 0}\left\|u_{t}(s)\right\|_{L^{2}}$ and $\lim _{s \rightarrow 0}\left\|\varphi_{t}(s)\right\|_{L^{2}}$. Recall that $\varepsilon_{1}=O\left(\varepsilon k_{\varepsilon}^{2}\right)$, the assertion (i) then follows from applying the Gronwall's lemma and Theorem 2.3.

(ii) First, testing (1.6) by $-\beta \varepsilon \Delta \varphi$ and using (2.13) we get

$$
\beta^{2} \varepsilon^{2}\|\Delta \varphi\|_{L^{2}}^{2} \leq\left\|\varphi_{t}\right\|_{L^{2}}^{2}+\alpha^{2}\|\nabla u\|_{L^{4}}^{4} \leq\left\|\varphi_{t}\right\|_{L^{2}}^{2}+\frac{\alpha^{2} \varepsilon_{1} C}{k_{\varepsilon}}\|\Delta u\|_{L^{2}}^{2}+\frac{\alpha^{2} \varepsilon_{1} C}{k_{\varepsilon}^{2} R_{1}^{2}} A T_{\varepsilon}\left(u_{0}, \varphi_{0}\right) .
$$

Then, testing (1.5) by $-\alpha k_{\varepsilon} \Delta u$ and using (2.12) and (2.13) we obtain

$$
\begin{aligned}
\alpha^{2} k_{\varepsilon}\left\|\sqrt{k_{\varepsilon}+\varphi^{2}} \Delta u\right\|_{L^{2}}^{2} \leq & \left\|u_{t}\right\|_{L^{2}}^{2}+\alpha^{2} k_{\varepsilon}\left(\|\nabla u\|_{L^{4}}^{4}+\|\nabla \varphi\|_{L^{4}}^{4}\right) \\
\leq & \left\|u_{t}\right\|_{L^{2}}^{2}+\frac{\alpha^{2} \varepsilon_{1} k_{\varepsilon} C}{\beta \varepsilon}\|\Delta \varphi\|_{L^{2}}^{2}+\frac{\alpha^{2} \varepsilon_{1}\left(1+k_{\varepsilon}\right) C}{k_{\varepsilon}}\|\Delta u\|_{L^{2}}^{2} \\
& +\left[\frac{\alpha^{2} \varepsilon_{1}\left(\beta^{2} \varepsilon^{2}+k_{\varepsilon}^{3}\right) C}{\beta^{2} \varepsilon^{2} k_{\varepsilon}^{2} R_{1}^{2}}\right] A T_{\varepsilon}\left(u_{0}, \varphi_{0}\right) .
\end{aligned}
$$

(iii) From (2.26) we have

$$
\left\|u_{t t}\right\|_{H^{-1}}^{2} \leq 2 \alpha\left[\left\|\sqrt{k_{\varepsilon}+\varphi^{2}} \nabla u_{t}\right\|_{L^{2}}^{2}+\left\|\varphi_{t} \nabla u\right\|_{L^{2}}^{2}\right]+2\|\gamma\|_{L^{\infty}}^{2}\left\|u_{t}\right\|_{L^{2}}^{2} .
$$

The assertion follows from integrating the above inequality in $t$ from 0 to $T_{1}$ and using (i) and (2.6).

(iv) From (2.27), the assertions (i)-(ii) and (2.6) we conclude

$$
\begin{aligned}
\int_{0}^{T_{1}}\left\|\varphi_{t t}\right\|_{H^{-1}}^{2} \mathrm{~d} s & \leq 8 \int_{0}^{T_{1}}\left[\beta^{2} \varepsilon^{2}\left\|\nabla \varphi_{t}\right\|_{L^{2}}^{2}+\alpha\left\|\nabla u \cdot \nabla u_{t} \varphi\right\|_{H^{-1}}^{2}+\alpha^{2}\left\||\nabla u|^{2} \varphi_{t}\right\|_{H^{-1}}^{2}+\frac{\beta^{2}}{\varepsilon^{2}}\left\|\varphi_{t}\right\|_{L^{2}}^{2}\right] \mathrm{d} s \\
& \leq 8 C_{1} \beta\left(\varepsilon+\frac{\beta T_{1}}{\varepsilon^{2}}\right)+C \alpha^{2} \int_{0}^{T_{1}}\|\nabla u\|_{L^{4}}^{2}\left[\left\|\varphi \nabla u_{t}\right\|_{L^{2}}^{2}+\left\|\nabla u \varphi_{t}\right\|_{L^{2}}^{2}\right] \mathrm{d} s \\
& \leq 8 C_{1} \beta\left(\varepsilon+\frac{\beta k_{\varepsilon}^{2}}{\varepsilon}\right)+C \alpha \int_{0}^{T_{1}}\|\Delta u\|_{L^{2}}\|\nabla u\|_{L^{2}}\left[\alpha\left\|\varphi \nabla u_{t}\right\|_{L^{2}}^{2}+\alpha\left\|\nabla u \varphi_{t}\right\|_{L^{2}}^{2}\right] \mathrm{d} s \\
& \leq 8 C_{1} \beta\left(\varepsilon+\beta k_{\varepsilon}\right)+\frac{C \sqrt{C_{2} A T_{\varepsilon}\left(u_{0}, \varphi_{0}\right)}}{\sqrt{\alpha} k_{\varepsilon}^{\frac{3}{2}}} C_{1} .
\end{aligned}
$$

The proof is complete.

Remark 2.4. We note that by Morrey's lemma, a maximum radius $R_{1}=R_{1}\left(\varepsilon, k_{\varepsilon} ; \alpha, \beta\right)$ in $(2.8)$ can be computed explicitly for a given $\varepsilon_{1}$ when the initial data $\left(u_{0}, \varphi_{0}\right) \in\left[H^{2}(\Omega)\right]^{2}$. 
The local strong solution exists until the first time $T=t_{1}$ such that

$$
\lim \sup _{t \rightarrow T}\left(A T_{\varepsilon}\left((u(t), \varphi(t)) ; B_{R}\left(x_{0}\right)\right)\right) \geq \varepsilon_{1} \quad \forall R>0 .
$$

By Theorem 2.2, $u_{t}, \varphi_{t} \in L^{2}\left(\Omega_{T}\right)$, hence the $L^{2}$-limits $u_{1}=\lim _{t \rightarrow T} u(t)$ and $\varphi_{1}=\lim _{t \rightarrow T} \varphi(t)$ exist. Let $(v, \psi)$ be a local strong solution of $(1.5)-(1.8)$ over the time interval $[T, \bar{T})$ with initial data $\left(u_{1}, \varphi_{1}\right)$ at time $T$. The composed function

$$
(\tilde{u}(t), \tilde{\varphi}(t))= \begin{cases}(u(t), \varphi(t)) & \text { for } 0 \leq t \leq T \\ (v(t), \psi(t)) & \text { for } T \leq t \leq \bar{T}\end{cases}
$$

then actually is a quasi-strong solution of $(1.5)-(1.8)$ over the time interval $[0, \bar{T}]$. It is easy to see that this extension process can be continued until $\bar{T}=\infty$.

We now prove that there are at most finite number of restarts in the above extension process. Moreover, we show that such a "piecewise" (in $t$ ) strong solution has at most finite number of point singularities in space-time where the solution ceases to be strong, and it is unique in this class. To state the result, we need the following notation

$$
\mathcal{Y}^{T}(\Omega):=\left\{\chi \in L^{\infty}\left((0, T) ; H^{1}(\Omega)\right): \exists\left(t_{k}, x_{k}\right)_{k=1}^{K}: \Delta \chi \in L^{2}\left(\Omega_{T} \backslash \bigcup_{1 \leq k \leq K} \overline{B_{\delta}\left(t_{k}, x_{k}\right)}\right), \forall \delta>0\right\}
$$

Theorem 2.4. Let $N=2$ and $T=\infty$. Suppose $A T_{\varepsilon}\left(u_{0}, \varphi_{0}\right)<\infty$ and $g \in L^{2}(\Omega)$. Then, there exist at most finite number space-time points $\left(x_{k}, t_{k}\right), 1 \leq k \leq K$ such that $(1.5)-(1.8)$ has a unique solution in $\left[\mathcal{Y}^{T}(\Omega)\right]^{2}$.

Proof. Since existence has been showed above, it suffices to prove that the singularity set must be finite in space-time and uniqueness. The idea of the proof is borrowed from [35].

Let $\bar{t}=\bar{t}_{1}$ be the first singular time, and

$$
\operatorname{Sing}(\bar{t})=\left\{x_{0} \in \Omega: \forall R>0: \lim \sup _{t \rightarrow \bar{t}} A T_{\varepsilon}\left((u(t), \varphi(t)) ; B_{R}\left(x_{0}\right)\right)>\varepsilon_{1}\right\}
$$

We want to show that $\operatorname{Sing}(\bar{t})$ is finite: suppose $x_{1}, \ldots, x_{K} \in \operatorname{Sing}(\bar{t})$. Choose $R>0$ such that $B_{2 R}\left(x_{i}\right) \cap$ $B_{2 R}\left(x_{j}\right)=\emptyset(i \neq j)$, and fix $\tau \in\left[\bar{t}-\frac{\varepsilon_{1} R^{2}}{2 \mathcal{B}_{2} A T\left(u_{0}, \varphi_{0}\right)}\right)$, where $\mathcal{B}_{2}$ is the constant in Lemma 2.1 and $\varepsilon_{1}=O\left(\varepsilon k_{\varepsilon}^{2}\right)$ same as in Theorem 2.2. According to Lemma 2.1,

$$
\begin{aligned}
K \varepsilon_{1} & \leq \sum_{k=1}^{K} \lim \sup _{t \rightarrow \bar{t}} A T_{\varepsilon}\left((u(t), \varphi(t)) ; B_{R}\left(x_{i}\right)\right) \\
& \leq \sum_{i=1}^{K}\left[A T_{\varepsilon}\left(u(\tau), \varphi(\tau) ; B_{2 R}\left(x_{i}\right)\right)+\frac{\varepsilon_{1}}{2}\right] \leq A T_{\varepsilon}\left(u_{0}, \varphi_{0}\right)+\frac{K \varepsilon_{1}}{2}
\end{aligned}
$$


and hence $K=K_{1}=\operatorname{card}\left(\operatorname{Sing}\left(\bar{t}_{1}\right)\right) \leq 2 A T_{\varepsilon}\left(u_{0}, \varphi_{0}\right) \varepsilon_{1}^{-1}$. Moreover, for $\left(u_{1}, \varphi_{1}\right)=\lim _{t \rightarrow \bar{t}_{1}}(u(t), \varphi(t))$ we have

$$
\begin{aligned}
A T_{\varepsilon}\left(u_{1}, \varphi_{1}\right) & =\lim _{R \rightarrow 0} A T_{\varepsilon}\left(\left(u_{1}, \varphi_{1}\right) ; \Omega \backslash \bigcup_{i=1}^{K_{1}} \overline{B_{2 R}\left(x_{i}\right)}\right) \\
& \leq \lim _{R \rightarrow 0} \lim _{t \rightarrow \bar{t}_{1}} A T_{\varepsilon}\left((u(t), \varphi(t)) ; \Omega \backslash \bigcup_{i=1}^{K_{1}} \overline{B_{2 R}\left(x_{i}\right)}\right) \\
& \leq \lim _{R \rightarrow 0} \lim _{t \rightarrow \bar{t}_{1}}\left[A T_{\varepsilon}(u(t), \varphi(t))-\sum_{i=1}^{K_{1}} A T_{\varepsilon}\left((u(t), \varphi(t)) ; B_{2 R}\left(x_{i}\right)\right)\right] \\
& \leq \lim _{R \rightarrow 0}\left[A T_{\varepsilon}\left(u_{0}, \varphi_{0}\right)-\lim _{t \rightarrow \bar{t}_{1}} \sum_{i=1}^{K_{1}} A T_{\varepsilon}\left((u(t), \varphi(t)) ; B_{2 R}\left(x_{i}\right)\right)\right] \\
& \leq A T_{\varepsilon}\left(u_{0}, \varphi_{0}\right)-\sum_{i=1}^{K_{1}} \lim _{R \rightarrow 0} \lim _{t \rightarrow \bar{t}_{1}} A T_{\varepsilon}\left((u(t), \varphi(t)) ; B_{R}\left(x_{i}\right)\right) \\
& \leq A T_{\varepsilon}\left(u_{0}, \varphi_{0}\right)-K_{1} \varepsilon_{1} .
\end{aligned}
$$

Similarly, let $K_{2}, K_{3}, \ldots$ be the number of singular points at consecutive times $\bar{t}_{2}<\bar{t}_{3}<\ldots$, and let $\left(u_{j}, \varphi_{j}\right)=$ $\lim _{t \rightarrow \bar{t}_{j}}(u(t), \varphi(t))$, for $j=2,3, \ldots$. By induction, we have

$$
A T_{\varepsilon}\left(u_{j}, \varphi_{j}\right) \leq A T_{\varepsilon}\left(u_{j-1}, \varphi_{j-1}\right)-K_{j} \varepsilon_{1} \leq \ldots \leq A T_{\varepsilon}\left(u_{0}, \varphi_{0}\right)-\left(K_{1}+\ldots+K_{j}\right) \varepsilon_{1} .
$$

This implies that the total number $K$ of singular points, hence also the number of singular times $\bar{t}_{j}$ is finite. Indeed, $K \leq A T_{\varepsilon}\left(u_{0}, \varepsilon_{0}\right) \varepsilon_{1}^{-1}$.

The finite number of singular times together with Theorem 2.1 implies uniqueness of solutions in the class $\mathcal{Y}^{T}$.

Remark 2.5. (a) It is not clear whether singularity really exists for a fixed $\varepsilon$, the above theorem describes the worst possible scenario when it does ${ }^{1}$.

(b) The estimate $K \leq A T_{\varepsilon}\left(u_{0}, \varepsilon_{0}\right) \varepsilon_{1}^{-1}$ indicates that $K$ could tend to infinity very fast as $\varepsilon \rightarrow 0$.

\section{AnAlysis of FUlly Discrete Finite ElEMENT APPROXimations}

In this section, we shall propose a family of fully discrete finite element discretizations for the gradient flow (1.5)-(1.8), including mass lumping strategies. Three (semi-)implicit schemes for both "consistent" and mass lumped spatial discretizations are analyzed in detail. We prove convergence of solutions to these discrete schemes ("mass lumped version") to weak and (piecewise) strong solutions of (1.5)-(1.8), and establish optimal order error estimates for the numerical solutions ("consistent version") in the case that (1.5)-(1.8) possesses strong solutions.

\subsection{Fully discrete schemes}

In the sequel, we assume that $\Omega \subset \mathbf{R}^{N}$ is a polygonal domain. Let $\mathcal{T}_{h}$ be a quasi-uniform triangulation of $\Omega$ ( $K \in \mathcal{T}_{h}$ are tetrahedrons when $N=3$ ) with mesh size $h \in(0,1)$. Let $V^{h}$ denote the finite element space of continuous, piecewise linear functions associated with $\mathcal{T}_{h}$, that is,

$$
V^{h}=V^{h}(\Omega):=\left\{v_{h} \in C^{0}(\bar{\Omega}) ;\left.v_{h}\right|_{K} \in P_{1}(K), \forall K \in \mathcal{T}_{h}\right\}
$$

\footnotetext{
${ }^{1} \mathrm{~A}$ recent discussion with John W. Barrett leads us to conclude that for each fixed $\varepsilon>0$, the singularity set of the solution to (1.5)-(1.8) is empty. This conclusion can be proved by using an argument of [5] where a similar parabolic system is studied.
} 
Let $\left\{t_{m}\right\}_{m=0}^{M}$ be an equidistant partition of $[0, T]$ of mesh size $k \in(0,1)$ and introduce the notation $d_{t} u^{m}:=$ $\left(u^{m}-u^{m-1}\right) / k$. We now define the following family of consistent fully discrete finite element discretizations for the gradient flow (1.5)-(1.8): Find $\left(u_{h}^{m}, \varphi_{h}^{m}\right) \in\left[V^{h}\right]^{2}$ for $m=0,1,2, \cdots, M$ such that

$$
\begin{aligned}
\left(d_{t} u_{h}^{m}, v_{h}\right)+\alpha\left(k_{\varepsilon} \nabla u_{h}^{m}+\left|\varphi_{h}^{m, \mu}\right|^{2} \nabla u_{h}^{m}, \nabla v_{h}\right)+\left(\gamma\left[u_{h}^{m}-g^{m}\right], v_{h}\right)=0 \quad \forall v_{h} \in V^{h}, \\
\left(d_{t} \varphi_{h}^{m}, \psi_{h}\right)+2 \beta \varepsilon\left(\nabla \varphi_{h}^{m}, \nabla \psi_{h}\right)+\alpha\left(\left|\nabla u_{h}^{m, \nu}\right|^{2} \varphi_{h}^{m}, \psi_{h}\right)+\frac{\beta}{2 \varepsilon}\left(\varphi_{h}^{m}-1, \psi_{h}\right)=0 \quad \forall \psi_{h} \in V^{h},
\end{aligned}
$$

with some starting value $\left(u_{h}^{0}, \varphi_{h}^{0}\right) \in\left[V^{h}\right]^{2}$.

Approximation of weak solutions requires a modification of this scheme ("mass lumped version") that has a maximum principle for discrete solutions $\left\{\varphi_{h}^{m}\right\}$ : find $\left(u_{h}^{m}, \varphi_{h}^{m}\right) \in\left[V^{h}\right]^{2}$ for $m=0,1,2, \cdots, M$ such that

$$
\begin{array}{cc}
\left(d_{t} u_{h}^{m}, v_{h}\right)+\alpha k_{\varepsilon}\left(\nabla u_{h}^{m}, \nabla v_{h}\right)+\alpha\left(\left|\varphi_{h}^{m, \mu}\right|^{2} \nabla u_{h}^{m}, \nabla v_{h}\right)_{h}+\left(\gamma\left[u_{h}^{m}-g^{m}\right], v_{h}\right)=0 \quad \forall v_{h} \in V^{h}, \\
\left(d_{t} \varphi_{h}^{m}, \psi_{h}\right)+2 \beta \varepsilon\left(\nabla \varphi_{h}^{m}, \nabla \psi_{h}\right)+\alpha\left(\left|\nabla u_{h}^{m, \nu}\right|^{2} \varphi_{h}^{m}, \psi_{h}\right)_{h}+\frac{\beta}{2 \varepsilon}\left(\varphi_{h}^{m}-1, \psi_{h}\right)_{h}=0 \quad \forall \psi_{h} \in V^{h},
\end{array}
$$

with some starting value $\left(u_{h}^{0}, \varphi_{h}^{0}\right) \in\left[V^{h}\right]^{2}$.

In both above discretizations, $(\cdot, \cdot)$ denotes the standard inner product of $L^{2}(\Omega)$, and

$$
\begin{array}{rlrl}
\varphi_{h}^{m, \mu} & :=\mu \varphi^{m}+(1-\mu) \varphi^{m-1} & & \forall \mu \in[0,1], \\
u_{h}^{m, \nu}:=\nu u^{m}+(1-\nu) u^{m-1} & \forall \nu \in[0,1] .
\end{array}
$$

Moreover, lumped mass integration in (3.3)-(3.4) is defined by

$$
(\chi, \eta)_{h}=\sum_{K \in \mathcal{T}_{h}} \int_{K} I_{h}(\chi \eta) \mathrm{d} x \quad \forall \chi, \eta \in C(\bar{\Omega})
$$

where $I_{h}: C(\bar{\Omega}) \rightarrow V^{h}$ denotes the (linear) Lagrange interpolation operator on $\mathcal{T}_{h}$. We recall the following properties of lumped mass integration (cf. Appendix of [20]),

$$
\begin{array}{rlrl}
\left|\left(\chi_{h}, \eta_{h}\right)_{h}-\left(\chi_{h}, \eta_{h}\right)\right| & \leq C h^{2}\left\|\chi_{h}\right\|_{H^{1}}\left\|\eta_{h}\right\|_{H^{1}} & & \forall \chi_{h}, \eta_{h} \in V^{h}, \\
\left|\left(\chi_{h}, \eta\right)_{h}-\left(\chi_{h}, \eta\right)\right| & \leq C h^{2}\left\|\chi_{h}\right\|_{H^{1}}\|\eta\|_{H^{2}} & & \forall \chi_{h} \in V^{h}, \quad \forall \eta \in H^{2}(\Omega), \\
c_{0}\left\|\chi_{h}\right\|_{L^{2}} \leq\left\|\chi_{h}\right\|_{h}:=\left(\left(\chi_{h}, \chi_{h}\right)_{h}\right)^{\frac{1}{2}} \leq c_{1}\left\|\chi_{h}\right\|_{L^{2}} & & \forall \chi_{h} \in V^{h},
\end{array}
$$

where $c_{i}>0, i=0,1$ does not depend on $h$.

Remark 3.1. If $\mu \nu \neq 0$, the above family of schemes is fully implicit, and if $\mu \nu=0$, the family is semiimplicit. In the rest of this section, we will mainly consider the following cases of $(\mu, \nu)$. (i) $(\mu, \nu)=(0,1)$, (ii) $(\mu, \nu)=(1,0)$, and (iii) $(\mu, \nu)=(1,1)$. We also remark that the unique solvability of $(3.1)-(3.2)$, and (3.3)-(3.4) is trivial for the cases (i) and (ii). For the case (iii), it can be shown by using a fixed point argumentation in view of the a priori estimates to be given in Lemma 3.1 below.

\subsection{Convergence of the fully discrete schemes}

In this section we shall establish convergence of a subsequence (resp. the whole sequence) of the fully discrete solution defined by (3.3)-(3.4) to a weak solution (resp. the strong solution) of (1.5)-(1.8) as $h, k \rightarrow 0$. We begin the subsection with a definition of acute triangulations [15], which will be imposed to ensure that the discrete maximum principle holds for the solution $\left\{\varphi_{h}^{m}\right\}$.

Definition 3.1. A triangulation $\mathcal{T}_{h}$ is said to be an acute triangulation if there exists $\theta_{0}>0$ such that every interior angle of all elements in $\mathcal{T}_{h}$ is less than or equal to $\frac{\pi}{2}-\theta_{0}$. 
Lemma 3.1. The solutions $\left(u_{h}^{m}, \varphi_{h}^{m}\right)$ of both (3.1)-(3.2) and (3.3)-(3.4) satisfy the following estimates:

$$
\begin{gathered}
\max _{0 \leq m \leq M}\left\|u_{h}^{m}\right\|_{L^{2}}^{2}+k \sum_{m=1}^{M}\left\{k\left\|d_{t} u_{h}^{m}\right\|_{L^{2}}^{2}+\alpha k_{\varepsilon}\left\|\nabla u_{h}^{m}\right\|_{L^{2}}^{2}+\alpha\left\|\varphi^{m, \mu} \nabla u_{h}^{m}\right\|_{h}^{2}\right. \\
\left.+\alpha c_{0}^{2}\left\|\varphi^{m, \mu} \nabla u_{h}^{m}\right\|_{L^{2}}^{2}+\left\|\sqrt{\gamma} u_{h}^{m}\right\|_{L^{2}}^{2}\right\} \leq\left\|u_{h}^{0}\right\|_{L^{2}}^{2}+k \sum_{m=1}^{M}\left\|\sqrt{\gamma} g^{m}\right\|_{L^{2}}^{2}, \\
\max _{0 \leq m \leq M}\left\|\varphi_{h}^{m}\right\|_{L^{2}}^{2}+k \sum_{m=1}^{M}\left\{k\left\|d_{t} \varphi_{h}^{m}\right\|_{L^{2}}^{2}+4 \beta \varepsilon\left\|\nabla \varphi_{h}^{m}\right\|_{L^{2}}^{2}+\alpha\left\|\varphi_{h}^{m} \nabla u^{m, \nu}\right\|_{h}^{2}\right. \\
\left.+\alpha c_{0}^{2}\left\|\varphi_{h}^{m} \nabla u^{m, \nu}\right\|_{L^{2}}^{2}+\frac{\beta}{2 \varepsilon}\left\|\varphi_{h}^{m}-1\right\|_{L^{2}}^{2}\right\} \leq\left\|\varphi_{h}^{0}\right\|_{L^{2}}^{2}+\frac{\beta T}{2 \varepsilon}|\Omega| .
\end{gathered}
$$

In the case (3.3)-(3.4), and for an acute triangulation $\mathcal{T}_{h}$ of $\Omega, h^{2} \leq C_{\theta_{0}} \beta \varepsilon k$, and $0 \leq \varphi_{h}^{0} \leq 1$, then

$$
0 \leq \varphi_{h}^{m} \leq 1 \quad \forall 0 \leq m \leq M \text {, a.e. } x \in \Omega .
$$

Where $C_{\theta_{0}}$ is positive constant which depends on $\theta_{0}$ but is independent of $h$.

Proof. The proof for $(3.1,3.2)$ is in the same line as that for Theorem 2.1. (3.9) and (3.10) are obtained by testing (3.1) by $u_{h}^{m}$ and testing (3.2) by $\varphi_{h}^{m}$, respectively, and applying the operator $k \sum_{m=1}^{\ell}(\ell \leq M)$ to the resulting inequalities. For $(3.3,3.4)$, since $I_{h}$ is linear and $\left|\nabla u_{h}^{m}\right|_{K} \equiv$ const for any $K \in \mathcal{T}_{h}$, elementwise application of (3.8) then yields assertions (3.9) and (3.10), respectively.

To show (3.11), let $\left\{\phi_{i}\right\}_{i=1}^{I}$ denote the canonical basis of $V^{h}, K:=\left\{k_{i j}\right\}_{i, j=1}^{I}$ be the stiffness matrix, $M:=\left\{m_{i j}\right\}_{i, j=1}^{I}$ the mass matrix, $M^{h}:=\left\{m_{i j}^{h}\right\}_{i, j=1}^{I}$ the lumped mass matrix, and $M_{\sigma}^{h}=\left\{\left(m_{\sigma}^{h}\right)_{i j}\right\}_{i, j=1}^{I}$, $\sigma \in \mathbb{R}^{I}$ a scaled lumped mass matrix, that is

$$
k_{i j}:=\left(\nabla \phi_{i}, \nabla \phi_{j}\right), \quad m_{i j}:=\left(\phi_{i}, \phi_{j}\right), \quad m_{i j}^{h}=\left(\phi_{i}, \phi_{j}\right)_{h}=\int_{\Omega} I_{h}\left(\phi_{i} \phi_{j}\right) \mathrm{d} x, \quad\left(m_{\sigma}^{h}\right)_{i j}=\left|\sigma_{i}\right|^{2} m_{i j}^{h}
$$

Consider $a_{i j}:=\frac{1}{k} m_{i j}+\beta \varepsilon k_{i j}+\alpha\left(m_{\nabla u_{h}^{m, \nu}}^{h}\right)_{i j}+\frac{\beta}{2 \varepsilon} m_{i j}^{h}$. Since $\mathcal{T}_{h}$ is acute, there exists $C_{\theta_{0}}>0$, such that $k_{i j} \leq-C_{\theta_{0}}<0$ uniformly in $h$, for any pair of adjacent nodes. This implies $a_{i j} \leq 0$, provided $i \neq j$ and $0 \leq k^{-1} \leq C_{\theta_{0}} h^{-2} ;\left\{a_{i j}\right\}_{i, j=1}^{I}$ is thus an $M$-matrix.

Now, the desired discrete maximum principle follows from testing (3.2) by $\left[\varphi_{h}^{m}\right]^{-}:=I_{h}\left(\varphi_{h}^{m}\right)^{-}$and $\left[\varphi_{h}^{m}-1\right]^{+}:=$ $I_{h}\left(\varphi_{h}^{m}-1\right)^{+}$, as well as utilizing an inductive argument used in the proofs of Lemmas 4.1 and 4.2 of [33].

We introduce the following modified energy $\overline{A T}_{\varepsilon}: V^{h} \times V^{h} \rightarrow \mathbf{R}$,

$$
\overline{A T}_{\varepsilon}\left(u_{h}^{m}, \varphi_{h}^{m}\right)=\frac{\alpha}{2}\left(k_{\varepsilon}\left\|\nabla u_{h}^{m}\right\|_{L^{2}}^{2}+\left\|\varphi_{h}^{m} \nabla u_{h}^{m}\right\|_{h}^{2}\right)+\beta \varepsilon\left\|\nabla \varphi_{h}^{m}\right\|_{L^{2}}^{2}+\frac{\beta}{2 \varepsilon}\left\|\varphi^{m}-1\right\|_{h}^{2}+\frac{\gamma}{2}\left\|u_{h}^{m}-g\right\|_{L^{2}}^{2} .
$$

It follows from (3.8) that

$$
\min \left\{c_{0}, 1\right\} A T_{\varepsilon}\left(u_{h}^{m}, \varphi_{h}^{m}\right) \leq \overline{A T}_{\varepsilon}\left(u_{h}^{m}, \varphi_{h}^{m}\right) \leq \max \left\{c_{1}, 1\right\} A T_{\varepsilon}\left(u_{h}^{m}, \varphi_{h}^{m}\right) .
$$


Lemma 3.2. Suppose that $g$ is independent of $t$, then the solution $\left(u_{h}^{m}, \varphi_{h}^{m}\right)$ of (3.3)-(3.4) satisfies the following equations.

(i) For $(\mu, \nu)=(1,0)$ and $(\mu, \nu)=(0,1)$, there holds for all $1 \leq \ell \leq M$

$$
\begin{aligned}
\overline{A T}_{\varepsilon}\left(u_{h}^{\ell}, \varphi_{h}^{\ell}\right)+ & k \sum_{m=1}^{\ell}\left[\left\|d_{t} u_{h}^{m}\right\|_{L^{2}}^{2}+\left\|d_{t} \varphi_{h}^{m}\right\|_{L^{2}}^{2}\right]+\frac{k^{2}}{2} \sum_{m=1}^{\ell}\left[\alpha k_{\varepsilon}\left\|d_{t} \nabla u_{h}^{m}\right\|_{L^{2}}^{2}+\alpha\left\|\varphi^{m, \mu} d_{t} \nabla u_{h}^{m}\right\|_{h}^{2}\right. \\
& \left.+\left\|\sqrt{\gamma} d_{t} u_{h}^{m}\right\|_{L^{2}}^{2}+2 \beta \varepsilon\left\|d_{t} \nabla \varphi_{h}^{m}\right\|_{L^{2}}^{2}+\alpha\left\|\nabla u^{m, \nu} d_{t} \varphi_{h}^{m}\right\|_{h}^{2}+\frac{\beta}{2 \varepsilon}\left\|d_{t} \varphi_{h}^{m}\right\|_{h}^{2}\right]=\overline{A T}_{\varepsilon}\left(u_{h}^{0}, \varphi_{h}^{0}\right) .
\end{aligned}
$$

(ii) For $(\mu, \nu)=(1,1)$, there holds for all $1 \leq \ell \leq M$

$$
\begin{aligned}
& \overline{A T}_{\varepsilon}\left(u_{h}^{\ell}, \varphi_{h}^{\ell}\right)+k \sum_{m=1}^{\ell}\left[\left\|d_{t} u_{h}^{m}\right\|_{L^{2}}^{2}+\left\|d_{t} \varphi_{h}^{m}\right\|_{L^{2}}^{2}\right]+\frac{k^{2}}{2} \sum_{m=1}^{\ell}\left[\alpha k_{\varepsilon}\left\|d_{t} \nabla u_{h}^{m}\right\|_{L^{2}}^{2}+\alpha\left\|\varphi^{m, \mu} d_{t} \nabla u_{h}^{m}\right\|_{h}^{2}+\left\|\sqrt{\gamma} d_{t} u_{h}^{m}\right\|_{L^{2}}^{2}\right. \\
& \left.\quad+2 \beta \varepsilon\left\|d_{t} \nabla \varphi_{h}^{m}\right\|_{L^{2}}^{2}+\alpha\left\|\nabla u^{m, \nu} d_{t} \varphi_{h}^{m}\right\|_{h}^{2}+\frac{\beta}{2 \varepsilon}\left\|d_{t} \varphi_{h}^{m}\right\|_{h}^{2}+\alpha\left(d_{t}\left|\varphi_{h}^{m}\right|^{2}, d_{t}\left|\nabla u_{h}^{m}\right|^{2}\right)_{h}\right]=\overline{A T}_{\varepsilon}\left(u_{h}^{0}, \varphi_{h}^{0}\right)
\end{aligned}
$$

Moreover, statements (i) and (ii) also hold for the solution of (3.1)-(3.2) with $A T_{\varepsilon}(\cdot, \cdot)$ and $\|\cdot\|_{L^{2}}$ being in place of $\overline{A T}_{\varepsilon}(\cdot, \cdot)$ and $\|\cdot\|_{h}$, respectively.

Proof. The proof of (3.14) is in the same line as that of (2.6). That is, the assertion follows from testing (3.3) by $d_{t} u_{h}^{m}$ and (3.4) by $d_{t} \varphi_{h}^{m}$, using the algebraic formulas

$$
\begin{aligned}
\left(d_{t} \xi^{m}\right) \xi^{m} & =\frac{1}{2} d_{t}\left|\xi^{m}\right|^{2}+\frac{k}{2}\left|d_{t} \xi^{m}\right|^{2}, \\
d_{t}\left(\xi^{m} \psi^{m}\right) & =\xi^{m} d_{t} \psi^{m}+\psi^{m-1} d_{t} \xi^{m}=\psi^{m} d_{t} \xi^{m}+\xi^{m-1} d_{t} \psi^{m}
\end{aligned}
$$

adding two resulting equations and applying the operator $k \sum_{m=1}^{\ell}(\ell \leq M)$ to the sum.

(3.15) follows from repeating the above procedure. The only difference is that we now need to use the following algebraic formula to handle the nonlinear terms

$$
\xi^{m} d_{t} \psi^{m}+\psi^{m} d_{t} \xi^{m}=d_{t}\left(\xi^{m} \psi^{m}\right)+k d_{t} \xi^{m} d_{t} \psi^{m}
$$

The proof is complete.

Remark 3.2. If $g \in L^{2}\left((0, T) ; L^{2}(\Omega)\right)$, similar estimates to (3.14) and (3.15) hold for $\left(u_{h}^{m}, \varphi_{h}^{m}\right)$. Since in this case we need to control the term $\left(g, d_{t} u_{h}^{m}\right)$ by $\frac{1}{2}\|g\|_{L^{2}}^{2}+\frac{1}{2}\left\|d_{t} u_{h}^{m}\right\|_{L^{2}}^{2}$, hence, we do not get equalities like (3.14) and (3.15), instead, we now obtain inequalities with a term involves $g^{m}$ on the right-hand sides.

Corresponding estimates to $(3.14)$ and $(3.15)$ for $(3.3,3.4)$ in terms of $A T_{\varepsilon}(\cdot)$ and mesh-independent norms immediately follow from (3.13) and (3.8).

We remark that estimate (3.15) is not in a closed form, since the last term on the left-hand side does not have a fixed sign. In fact, it is not clear if that term can be absorbed by the positive terms that proceed it, allowing that $h, k$ and $\mathcal{T}_{h}$ satisfy some constraints. 
For the fully discrete finite element solutions $\left\{u_{h}^{m}\right\}$ and $\left\{\varphi_{h}^{m}\right\}$, we define their constant and linear interpolations in $t$ as follows:

$$
\begin{array}{lrr}
\bar{u}_{h}(\cdot, t):=u_{h}^{m}, \quad \underline{u}_{h}(\cdot, t):=u_{h}^{m-1} & \forall t \in\left(t_{m-1}, t_{m}\right), \quad 1 \leq m \leq M, \\
\overline{\bar{u}}_{h}(\cdot, t):=\frac{t-t_{m-1}}{k} u_{h}^{m}+\frac{t_{m}-t}{k} u_{h}^{m-1} \quad \forall t \in\left[t_{m-1}, t_{m}\right], \quad 1 \leq m \leq M ; \\
\bar{\varphi}_{h}(\cdot, t):=\varphi_{h}^{m}, \quad \underline{\varphi}_{h}(\cdot, t):=\varphi_{h}^{m-1} \quad \forall t \in\left(t_{m-1}, t_{m}\right), \quad 1 \leq m \leq M, \\
\overline{\bar{\varphi}}_{h}(\cdot, t):=\frac{t-t_{m-1}}{k} \varphi_{h}^{m}+\frac{t_{m}-t}{k} \varphi_{h}^{m-1} & \forall t \in\left[t_{m-1}, t_{m}\right], \quad 1 \leq m \leq M, \\
\bar{u}_{h}(\cdot, 0)=\underline{u}_{h}(\cdot, 0)=\overline{\bar{u}}_{h}(\cdot, 0):=u_{h}^{0}, \quad \bar{\varphi}_{h}(\cdot, 0)=\underline{\varphi}_{h}(\cdot, 0)=\overline{\bar{\varphi}}_{h}(\cdot, 0):=\varphi_{h}^{0} .
\end{array}
$$

Clearly, $\bar{u}_{h}, \underline{u}_{h}, \bar{\varphi}_{h}$ and $\underline{\varphi}_{h}$ are continuous in $x$ but discontinuous in $t$. On the other hand, $\overline{\bar{u}}_{h}$ and $\overline{\bar{\varphi}}_{h}$ are continuous in both $x$ and $t$.

Theorem 3.1. Let $\left(u_{h}, \varphi_{h}\right)$ be the solution of (3.3)-(3.4), which is defined on an acute triangulation $\mathcal{T}_{h}$, and $h^{2} \leq C_{\theta_{0}} \beta \varepsilon k$. Suppose that the starting value $\left(u_{h}^{0}, \varphi_{h}^{0}\right)$ satisfies

$$
\lim _{h, k \rightarrow 0}\left(\left\|u_{h}^{0}-u_{0}\right\|_{L^{2}}+\left\|\varphi_{h}^{0}-\varphi_{0}\right\|_{L^{2}}\right)=0 .
$$

Let $(\mu, \nu)=(1,1)$. Then there exists a subsequence of $\left\{\left(u_{h}, \varphi_{h}\right)\right\}$ (still denoted by the same notation) and a solution $(u, \varphi)$ of (1.5)-(1.8) such that

$$
\begin{aligned}
& \lim _{h, k \rightarrow 0}\left(\left\|\bar{u}_{h}-u\right\|_{L^{2}\left(L^{2}\right)}+\left\|\bar{\varphi}_{h}-\varphi\right\|_{L^{2}\left(L^{2}\right)}\right)=0, \\
& \lim _{h, k \rightarrow 0}\left(\left\|\underline{u}_{h}-u\right\|_{L^{2}\left(L^{2}\right)}+\left\|\underline{\varphi}_{h}-\varphi\right\|_{L^{2}\left(L^{2}\right)}\right)=0, \\
& \lim _{h, k \rightarrow 0}\left(\left\|\overline{\bar{u}}_{h}-u\right\|_{L^{2}\left(L^{2}\right)}+\left\|\overline{\bar{\varphi}}_{h}-\varphi\right\|_{L^{2}\left(L^{2}\right)}\right)=0 .
\end{aligned}
$$

Proof. First we notice that (3.9)-(3.11) imply the following (uniform in $h$ and $k$ ) estimates:

$$
\begin{aligned}
& \left\|\nabla \overline{\bar{u}}_{h}\right\|_{L^{2}\left(L^{2}\right)} \leq 2\left\|\nabla \bar{u}_{h}\right\|_{L^{2}\left(L^{2}\right)}=2\left\|\nabla \underline{u}_{h}\right\|_{L^{2}\left(L^{2}\right)}=2\left(k \sum_{m=1}^{M}\left\|\nabla u_{h}^{m}\right\|_{L^{2}}^{2}\right)^{\frac{1}{2}} \leq \frac{C}{\sqrt{k_{\varepsilon}}} \\
& \left\|\overline{\bar{u}}_{h}\right\|_{L^{\infty}\left(L^{2}\right)} \leq\left\|\bar{u}_{h}\right\|_{L^{\infty}\left(L^{2}\right)}=\left\|\underline{u}_{h}\right\|_{L^{\infty}\left(L^{2}\right)}=\max _{0 \leq m \leq M}\left\|u_{h}^{m}\right\|_{L^{2}} \leq C, \\
& \sum_{i=1}^{M}\left\|u_{h}^{m}-u_{h}^{m-1}\right\|_{L^{2}}^{2} \leq C, \\
& \left\|\nabla \overline{\bar{\varphi}}_{h}\right\|_{L^{2}\left(L^{2}\right)} \leq 2\left\|\nabla \bar{\varphi}_{h}\right\|_{L^{2}\left(L^{2}\right)}=2\left\|\nabla \underline{\varphi}_{h}\right\|_{L^{2}\left(L^{2}\right)}=2\left(k \sum_{m=1}^{M}\left\|\nabla \varphi_{h}^{m}\right\|_{L^{2}}^{2}\right)^{\frac{1}{2}} \leq \frac{C}{\sqrt{\varepsilon}} \\
& 0 \leq \min _{0 \leq m \leq M} \varphi_{h}^{m} \leq \overline{\bar{\varphi}}_{h}(x, t), \bar{\varphi}_{h}(x, t), \underline{\varphi}_{h}(x, t) \leq \max _{0 \leq m \leq M} \varphi_{h}^{m} \leq 1 \quad(x, t) \in \Omega_{T}, \\
& \sum_{i=1}^{M}\left\|\varphi_{h}^{m}-\varphi_{h}^{m-1}\right\|_{L^{2}}^{2} \leq C .
\end{aligned}
$$


Then there exist convergent subsequences of $\left\{\bar{u}_{h}\right\}$ and $\left\{\bar{\varphi}_{h}\right\}$ (denoted by the same notation) and a pair of functions $(\hat{u}, \hat{\varphi}) \in\left[L^{\infty}\left((0, T) ; L^{2}(\Omega)\right) \cap L^{2}\left((0, T) ; H^{1}(\Omega)\right)\right]^{2}$ such that as $h, k \rightarrow 0$

$$
\begin{aligned}
& \bar{u}_{h} \longrightarrow \hat{u} \quad \text { weakly } \text { in } L^{\infty}\left((0, T) ; L^{2}(\Omega)\right), \\
& \text { weakly in } L^{2}\left((0, T) ; H^{1}(\Omega)\right) \\
& \bar{\varphi}_{h} \longrightarrow \hat{\varphi} \quad \text { weakly } \text { in } L^{\infty}\left(\Omega_{T}\right) \text {, } \\
& \text { weakly in } L^{2}\left((0, T) ; H^{1}(\Omega)\right) \text {. }
\end{aligned}
$$

Following a standard procedure (cf. [25]), it can be shown that

$$
\bar{u}_{h} \longrightarrow \hat{u}, \quad \bar{\varphi}_{h} \longrightarrow \hat{\varphi} \quad \text { strongly in } L^{2}\left(\Omega_{T}\right)
$$

by first proving $\left\{\left(\bar{u}_{h}, \bar{\varphi}_{h}\right)\right\}$ is a Cauchy sequence in $\left[L^{2}\left(\Omega_{T}\right)\right]^{2}$ for almost every $t \in[0, T]$. We remark that (3.21) is required in this step.

Hence, (3.33) implies that there exist subsequences of $\left\{\bar{u}_{h}\right\}$ and $\left\{\bar{\varphi}_{h}\right\}$ (still denoted by the same notation) such that

$$
\bar{u}_{h} \longrightarrow \hat{u}, \quad \bar{\varphi}_{h} \longrightarrow \hat{\varphi} \quad \text { a.e. in } \Omega_{T},
$$

which and (3.29) immediately imply

$$
0 \leq \hat{\varphi}(x, t) \leq 1 \quad \text { for a.e. }(x, t) \in \Omega_{T} .
$$

From (3.27) we have

$$
\begin{aligned}
\left\|\overline{\bar{u}}_{h}-\bar{u}_{h}\right\|_{L^{2}\left(L^{2}\right)}^{2} & =\int_{0}^{T}\left\|\overline{\bar{u}}_{h}-\bar{u}_{h}\right\|_{L^{2}}^{2} \mathrm{~d} t \\
& =\sum_{m=1}^{M}\left\|u_{h}^{m}-u_{h}^{m-1}\right\|_{L^{2}}^{2} \int_{t_{m-1}}^{t_{m}}\left(\frac{t_{m}-t}{k}\right)^{2} \mathrm{~d} t \\
& =\frac{k}{3} \sum_{m=1}^{M}\left\|u_{h}^{m}-u_{h}^{m-1}\right\|_{L^{2}}^{2} \leq C k .
\end{aligned}
$$

Similarly,

$$
\left\|\overline{\bar{u}}_{h}-\underline{u}_{h}\right\|_{L^{2}\left(L^{2}\right)}^{2}+\left\|\overline{\bar{\varphi}}_{h}-\bar{\varphi}_{h}\right\|_{L^{2}\left(L^{2}\right)}^{2}+\left\|\overline{\bar{\varphi}}_{h}-\underline{\varphi}_{h}\right\|_{L^{2}\left(L^{2}\right)}^{2} \leq C k .
$$

In view of $(3.33),(3.36,3.37)$, we conclude that $(3.22,3.24)$ hold provided that we can show $(\hat{u}, \hat{\varphi})$ is a weak solution of (1.5)-(1.8).

Let $\phi \in C_{0}^{\infty}((0, T))$ and $(v, \psi) \in\left[C^{\infty}(\Omega)\right]^{2}$. It follows from the finite element theory $[11,15]$ that there exists a sequence $\left\{\left(v_{h}, \psi_{h}\right)\right\} \in\left[V^{h}\right]^{2}$ which converges to $(v, \psi)$ strongly in $\left[W^{1, \infty}(\Omega)\right]^{2}$. Now multiply (3.3) and (3.4) 
by $\phi(t)$ and integrate in $t$ from 0 to $T$ to get

$$
\begin{gathered}
\int_{0}^{T}\left[\left(\overline{\bar{u}}_{h}(t), \phi^{\prime}(t) v_{h}\right)-\alpha\left(\left[k_{\varepsilon}+\left|\bar{\varphi}_{h}\right|^{2}\right] \nabla \bar{u}_{h}, \nabla\left(\phi(t) v_{h}\right)\right)-\left(\gamma\left[\bar{u}_{h}-\bar{g}_{h}\right], \phi(t) v_{h}\right)\right] \mathrm{d} t= \\
\int_{0}^{T} \int_{\Omega}\left(I_{h}\left[\left|\bar{\varphi}_{h}\right|^{2} \nabla \bar{u}_{h} \nabla\left(\phi(t) v_{h}\right)\right]-\left|\bar{\varphi}_{h}\right|^{2} \nabla \bar{u}_{h} \nabla\left(\phi(t) v_{h}\right)\right) \mathrm{d} x \mathrm{~d} t \\
\int_{0}^{T}\left[\left(\overline{\bar{\varphi}}_{h}, \phi^{\prime}(t) \psi_{h}\right)-2 \beta \varepsilon\left(\nabla \bar{\varphi}_{h}, \nabla\left(\phi(t) \psi_{h}\right)\right)-\alpha\left(\left|\nabla \bar{u}_{h}\right|^{2} \bar{\varphi}_{h}, \phi(t) \psi_{h}\right)-\frac{\beta}{2 \varepsilon}\left(\bar{\varphi}_{h}-1, \phi(t) \psi_{h}\right)\right] \mathrm{d} t= \\
\alpha \int_{0}^{T} \int_{\Omega}\left(I_{h}\left[\left|\nabla \bar{u}_{h}\right|^{2} \bar{\varphi}_{h} \phi(t) \psi_{h}\right]-\left|\nabla \bar{u}_{h}\right|^{2} \bar{\varphi}_{h} \phi(t) \psi_{h}\right) \mathrm{d} x \mathrm{~d} t+\frac{\beta}{2 \varepsilon} \int_{0}^{T} \int_{\Omega}\left(I_{h}\left[\left(\bar{\varphi}_{h}-1\right) \phi(t) \psi_{h}\right]-\left(\bar{\varphi}_{h}-1\right) \phi(t) \psi_{h}\right) \mathrm{d} x \mathrm{~d} t,
\end{gathered}
$$

where $\bar{g}_{h}$ denotes the (upper) constant interpolation of $\left\{g^{m}\right\}$ in $t$ (cf. (3.16)).

Sending $h, k \rightarrow 0$ in the last two inequalities, it follows from (3.31)-(3.36) and a separability argument for $L^{2}\left((0, T) ; L^{1}(\Omega)\right)$ that

$$
\begin{array}{r}
\int_{0}^{T}\left[\left(\hat{u}(t), \phi^{\prime}(t) v\right)-\alpha\left(\left[k_{\varepsilon}-|\hat{\varphi}|^{2}\right] \nabla \hat{u}, \nabla(\phi(t) v)\right)-(\gamma[\hat{u}-g], \phi(t) v)\right] \mathrm{d} t=0, \\
\int_{0}^{T}\left[\left(\hat{\varphi}, \phi^{\prime}(t) \psi\right)-2 \beta \varepsilon(\nabla \hat{\varphi}, \nabla(\phi(t) \psi))-\alpha\left(|\nabla \hat{u}|^{2} \hat{\varphi}, \phi(t) \psi\right)+\frac{\beta}{2 \varepsilon}(\hat{\varphi}-1, \phi(t) \psi)\right] \mathrm{d} t=0,
\end{array}
$$

that is, $(\hat{u}, \hat{\varphi})$ satisfies $(1.5)-(1.7)$ in the distribution sense.

Finally, it remains to show $\hat{u}(0)=u_{0}$ and $\hat{\varphi}(0)=\varphi_{0}$. This follows the same guidelines. The main difference is that now we choose $\phi \in C^{\infty}([0, T])$ such that $\phi(0)=1$ and $\phi(T)=0$, and use the integration by parts formulas

$$
\begin{aligned}
& \int_{0}^{T}\left(\overline{\bar{u}}_{h}^{\prime}(t), \phi(t) v_{h}\right) \mathrm{d} t=-\int_{0}^{T}\left(\overline{\bar{u}}_{h}(t), \phi^{\prime}(t) v_{h}\right) \mathrm{d} t+\left(u_{h}^{0}, \phi(0) v_{h}\right), \\
& \int_{0}^{T}\left(\overline{\bar{\varphi}}_{h}^{\prime}(t), \phi(t) v_{h}\right) \mathrm{d} t=-\int_{0}^{T}\left(\overline{\bar{\varphi}}_{h}(t), \phi^{\prime}(t) v_{h}\right) \mathrm{d} t+\left(\varphi_{h}^{0}, \phi(0) v_{h}\right)
\end{aligned}
$$

and (3.21). The proof is complete.

For the cases $(\mu, \nu)=(1,0)$ and $(\mu, \nu)=(0,1)$, to show that $\left(\bar{u}_{h}, \bar{\varphi}_{h}\right)$ has a subsequence satisfying $(3.22)-$ (3.24), we need to use the stronger estimate (3.14) in order to ensure $\nabla \overline{\bar{u}}_{h}, \nabla \bar{u}_{h}$ and $\nabla \underline{u}_{h}$, respectively, $\nabla \overline{\bar{\varphi}}_{h}, \nabla \bar{\varphi}_{h}$ and $\nabla \underline{\varphi}_{h}$ converge (weakly) to the same limit.

Theorem 3.2. Let $\left(u_{h}, \varphi_{h}\right)$ be the solution of (3.3)-(3.4) which is defined on an acute triangulation $\mathcal{T}_{h}$, and $h^{2} \leq C_{\theta_{0}} \beta \varepsilon k$. Suppose that the starting value $\left(u_{h}^{0}, \varphi_{h}^{0}\right)$ satisfies (3.21) and the constraint $A T_{\varepsilon}\left(u_{h}^{0}, \varphi_{h}^{0}\right)<\infty$. Then, the conclusions of Theorem 3.1 hold for the semi-implicit schemes corresponding to $(\mu, \nu)=(1,0)$ and $(\mu, \nu)=(0,1)$.

Proof. Since the proof follows the exact same lines as that of Theorem 3.1, we only sketch the main differences. 
In addition to the estimates (3.25)-(3.30), from (3.14) we also get

$$
\begin{aligned}
& \left\|\left(\overline{\bar{u}}_{h}\right)_{t}\right\|_{L^{2}\left(L^{2}\right)}=\left(k \sum_{m=1}^{M}\left\|d_{t} u_{h}^{m}\right\|_{L^{2}}^{2}\right)^{\frac{1}{2}} \leq C, \\
& \left\|\nabla \overline{\bar{u}}_{h}\right\|_{L^{\infty}\left(L^{2}\right)} \leq\left\|\nabla \bar{u}_{h}\right\|_{L^{\infty}\left(L^{2}\right)}=\left\|\nabla \underline{u}_{h}\right\|_{L^{\infty}\left(L^{2}\right)}=\max _{0 \leq m \leq M}\left\|\nabla u_{h}^{m}\right\|_{L^{2}} \leq \frac{C}{\sqrt{k_{\varepsilon}}} \\
& \sum_{i=1}^{M}\left\|\nabla u_{h}^{m}-\nabla u_{h}^{m-1}\right\|_{L^{2}}^{2} \leq \frac{C}{\sqrt{k_{\varepsilon}}}, \\
& \left\|\left(\overline{\bar{\varphi}}_{h}\right)_{t}\right\|_{L^{2}\left(L^{2}\right)}=\left(k \sum_{m=1}^{M}\left\|d_{t} \varphi_{h}^{m}\right\|_{L^{2}}^{2}\right)^{\frac{1}{2}} \leq C, \\
& \left\|\nabla \overline{\bar{\varphi}}_{h}\right\|_{L^{\infty}\left(L^{2}\right)} \leq\left\|\nabla \bar{\varphi}_{h}\right\|_{L^{\infty}\left(L^{2}\right)}=\left\|\nabla \underline{\varphi}_{h}\right\|_{L^{\infty}\left(L^{2}\right)}=\max _{0 \leq m \leq M}\left\|\nabla \varphi_{h}^{m}\right\|_{L^{2}} \leq \frac{C}{\sqrt{\varepsilon}} \\
& \sum_{i=1}^{M}\left\|\nabla \varphi_{h}^{m}-\nabla \varphi_{h}^{m-1}\right\|_{L^{2}}^{2} \leq \frac{C}{\sqrt{\varepsilon}} .
\end{aligned}
$$

From (3.40) we have

$$
\begin{aligned}
\left\|\nabla \overline{\bar{u}}_{h}-\nabla \bar{u}_{h}\right\|_{L^{2}\left(L^{2}\right)}^{2} & =\int_{0}^{T}\left\|\nabla \overline{\bar{u}}_{h}-\nabla \bar{u}_{h}\right\|_{L^{2}}^{2} \mathrm{~d} t \\
& =\sum_{m=1}^{M}\left\|\nabla u_{h}^{m}-\nabla u_{h}^{m-1}\right\|_{L^{2}}^{2} \int_{t_{m-1}}^{t_{m}}\left(\frac{t_{m}-t}{k}\right)^{2} \mathrm{~d} t \\
& =\frac{k}{3} \sum_{m=1}^{M}\left\|\nabla u_{h}^{m}-\nabla u_{h}^{m-1}\right\|_{L^{2}}^{2} \leq \frac{C k}{\sqrt{k_{\varepsilon}}} .
\end{aligned}
$$

Similarly,

$$
\begin{aligned}
\left\|\nabla \overline{\bar{u}}_{h}-\nabla \underline{u}_{h}\right\|_{L^{2}\left(L^{2}\right)}^{2} \leq \frac{C k}{\sqrt{k_{\varepsilon}}} \\
\left\|\nabla \overline{\bar{\varphi}}_{h}-\nabla \bar{\varphi}_{h}\right\|_{L^{2}\left(L^{2}\right)}^{2} \leq \frac{C k}{\sqrt{\varepsilon}} \\
\left\|\nabla \overline{\bar{\varphi}}_{h}-\nabla \underline{\varphi}_{h}\right\|_{L^{2}\left(L^{2}\right)}^{2} \leq \frac{C k}{\sqrt{\varepsilon}}
\end{aligned}
$$

The remaining part of the proof is to extract a convergent subsequence of $\left\{\left(u_{h}^{m}, \varphi_{h}^{m}\right)\right\}$ (still denoted by the same notation) and then pass to the limit. Due to the stronger estimates in (3.38)-(3.47), it is easier to pass to the limits because now not only $\overline{\bar{u}}_{h}, \bar{u}_{h}$ and $\underline{u}_{h}$, respectively, $\bar{\varphi}_{h}, \bar{\varphi}_{h}$ and $\underline{\varphi}_{h}$, converge strongly to the same limit in $L^{2}\left(\Omega_{T}\right)$, but also their respective gradients converge weakly $\star$ to the same limit in $L^{\infty}\left((0, T) ; H^{1}(\Omega)\right)$. Also, in view of (3.38) and (3.41), (3.33) follows directly from applying an Aubin-Lions compactness lemma [27,34]. $\square$ have

For $\left(u_{0}, \varphi_{0}\right) \in\left[H^{1}(\Omega)\right]^{2}$, since (piecewise) strong solutions are unique in the sense of Theorem 2.4, then we

Corollary 3.1. Suppose $A T_{\varepsilon}\left(u_{0}, \varphi_{0}\right)<\infty$ and $g \in L^{2}(\Omega)$. Let $\mathcal{T}_{h}$ be an acute triangulation of $\Omega$, and $h^{2} \leq$ $C_{\theta_{0}} \beta \varepsilon k$ in scheme (3.3)-(3.4). Then, the whole sequence $\left\{\left(u_{h}, \varphi_{h}\right)\right\}$ of each of the fully implicit and semiimplicit finite element solutions satisfies (3.22)-(3.24). 
Remark 3.3. We conclude this subsection by commenting on the choices of the starting value $\left(u_{h}^{0}, \varphi_{h}^{0}\right)$ for the fully discrete finite element schemes in (3.3)-(3.4). It follows from [15] that if we set $u_{h}^{0}=Q_{h} u_{0}$ and $\varphi_{h}^{0}=Q_{h} \varphi_{0}$, the $L^{2}$-projections of $u_{0}$ and $\varphi_{0}$ in $V^{h}$, respectively, then all the requirements of Theorems 3.1 and 3.2 for $\left(u_{h}^{0}, \varphi_{h}^{0}\right)$ are satisfied. We also note that $u_{h}^{0}=I_{h} u_{0}$ and $\varphi_{h}^{0}=I_{h} \varphi_{0}$, the piecewise linear interpolations of $u_{0}$ and $\varphi_{0}$ into $V^{h}$, satisfy the constraints of Theorem 3.1, but may fail to satisfy the requirement "AT $\left(I_{h} u^{0}, I_{h} \varphi^{0}\right)<\infty$ uniformly in $h$ " of Theorem 3.2.

\subsection{Error estimates for the fully discrete schemes}

In this last section, we shall derive some error estimates for the fully discrete finite element solutions defined in (3.1)-(3.2) in the two dimensional case (i.e. $N=2)$, provided that the solution $(u, \varphi)$ of $(1.5)-(1.8)$ belongs to $\left[L^{\infty}\left((0, T) ; W^{1, \infty}(\Omega)\right) \cap L^{2}\left((0, T) ; H^{2}(\Omega)\right) \cap H^{2}\left((0, T) ; H^{-1}(\Omega)\right)\right]^{2}$ satisfying assumption (3.54) below. The analysis relies on regularity of the strong solution $(u, \varphi)$ and thus allows to use (3.1)-(3.2) rather than its lumped version which satisfies a discrete maximum principle.

We establish practically useful error estimates which depend on $\varepsilon^{-1}$ and $k_{\varepsilon}^{-1}$ in some low polynomial order. Our analysis also carries over to solutions in $\left[L^{2}\left((0, T) ; H^{2}(\Omega)\right) \cap H^{2}\left((0, T) ; H^{-1}(\Omega)\right)\right]^{2}$ as well, at the expense that error constant depends exponentially on $\varepsilon^{-1}$ and $k_{\varepsilon}^{-1}$ (cf. (e) of Rem. 3.4).

We begin with definitions of two projection operators. First, let $Q_{h}: L^{2}(\Omega) \rightarrow V^{h}$ denote the $L^{2}$ projection operator

$$
\left(w-Q_{h} w, v_{h}\right)=0 \quad \forall v_{h} \in V^{h} .
$$

Second, let $P_{h}: H^{1}(\Omega) \rightarrow V^{h} \cap H_{0}^{1}(\Omega)$ denote the usual elliptic projection operator

$$
\left(\nabla\left(w-P_{h} w\right), \nabla v_{h}\right)=0 \quad \forall v_{h} \in V^{h} \cap H_{0}^{1}(\Omega) .
$$

It is well-known that the above projection operators have the following approximation properties $[11,15]$.

Lemma 3.3. There exists a positive constant $C$ such that for $h \in(0,1)$

$$
\begin{aligned}
& \left\|v-Q_{h} v\right\|_{L^{2}}+h\left\|\nabla\left(v-Q_{h} v\right)\right\|_{L^{2}} \leq C h\|v\|_{H^{1}} \quad \forall v \in H^{1}(\Omega), \\
& \left\|v-Q_{h} v\right\|_{L^{2}} \leq C h^{2}\|v\|_{H^{2}} \quad \forall v \in H^{2}(\Omega), \\
& \left\|v-P_{h} v\right\|_{L^{2}}+h\left\|\nabla\left(v-P_{h} v\right)\right\|_{L^{2}} \leq C h^{2}\|v\|_{H^{2}} \quad \forall v \in H^{2}(\Omega), \\
& \left\|v-P_{h} v\right\|_{L^{\infty}} \leq C h|\ln h|\|\nabla v\|_{L^{\infty}} \quad \forall v \in W^{1, \infty}(\Omega) .
\end{aligned}
$$

Theorem 3.3. Let $\left(u_{0}, \varphi_{0}\right) \in\left[H^{2}(\Omega)\right]^{2}$. Let $\mathcal{T}_{h}$ be a quasi-uniform triangulation of $\Omega$, and $(u, \varphi) \in$ $\left[L^{\infty}\left((0, T) ; W^{1, \infty}(\Omega)\right) \cap L^{2}\left((0, T) ; H^{2}(\Omega)\right) \cap H^{2}\left((0, T) ; H^{-1}(\Omega)\right)\right]^{2}$ satisfies the growth condition

$$
C^{*}:=\|\nabla u\|_{L^{\infty}\left(L^{\infty}\right)}+\|\nabla \varphi\|_{L^{\infty}\left(L^{\infty}\right)}=O\left(\varepsilon^{-\frac{1}{2}}\right) \quad(\text { for } \varepsilon \ll 1) .
$$

For each of three pairs of $(\mu, \nu)$ :

$$
\begin{aligned}
\text { (i) }(\mu, \nu) & =(0,1), \\
\text { (ii) }(\mu, \nu) & =(1,0), \\
\text { (iii) }(\mu, \nu) & =(1,1),
\end{aligned}
$$


in (3.1)-(3.2), let $E_{u}^{m}:=u\left(t_{m}\right)-u_{h}^{m} \quad$ and $E_{\varphi}^{m}:=\varphi\left(t_{m}\right)-\varphi_{h}^{m}$. Then, under the following starting value and mesh constraints

$$
\begin{aligned}
\left\|u_{h}^{0}-u_{0}\right\|_{L^{2}}+\left\|\varphi_{h}^{0}-\varphi_{0}\right\|_{L^{2}} & \leq C h, \\
h|\ln h| & <\frac{\alpha k_{\varepsilon}}{C^{*}}=O\left(\varepsilon^{\frac{1}{2}} k_{\varepsilon}\right), \\
k & =o\left(h^{\frac{1}{2}}\right),
\end{aligned}
$$

the global errors $E_{u}^{m}$ and $E_{\varphi}^{m}$ satisfy

$$
\begin{array}{r}
\max _{0 \leq m \leq M}\left[\left\|E_{u}^{m}\right\|_{L^{2}}\right. \\
\left.+\left\|E_{\varphi}^{m}\right\|_{L^{2}}\right]+\left\{k \sum _ { m = 1 } ^ { M } \left[k\left\|d_{t} E_{u}^{m}\right\|_{L^{2}}^{2}+k\left\|d_{t} E_{\varphi}^{m}\right\|_{L^{2}}^{2}+\alpha k_{\varepsilon}\left\|\nabla E_{u}^{m}\right\|_{L^{2}}^{2}+\alpha\left\|\varphi\left(t_{m}\right) \nabla E_{u}^{m}\right\|_{L^{2}}^{2}\right.\right. \\
\left.\left.+\left\|\sqrt{\gamma} E_{u}^{m}\right\|_{L^{2}}^{2}+\beta \varepsilon\left\|\nabla E_{\varphi}^{m}\right\|_{L^{2}}^{2}+\alpha\left\|\nabla u\left(t_{m}\right) E_{\varphi}^{m}\right\|_{L^{2}}^{2}+\frac{\beta}{\varepsilon}\left\|E_{\varphi}^{m}\right\|_{L^{2}}^{2}\right]\right\}^{\frac{1}{2}} \leq \hat{C}(h+k), \quad(3.58)
\end{array}
$$

where $\hat{C}$ is some positive constant which depends linearly on $\frac{1}{\sqrt{\varepsilon}}$ and $\frac{1}{\sqrt{k_{\varepsilon}}}$, and on $C^{*}$, and $C_{0}$ to $C_{4}$, which are defined in Theorem 2.3 and Corollary 2.1, in some low polynomial order.

Proof. We shall prove the above error estimate for the three fully discrete schemes corresponding to (i) $(\mu, \nu)=$ $(0,1)$, (ii) $(\mu, \nu)=(1,0)$, and (iii) $(\mu, \nu)=(1,1)$ together. For the purpose, we introduce the notation

$$
\begin{array}{ll}
\varphi^{m, \mu}:=\mu \varphi\left(t_{m}\right)+(1-\mu) \varphi\left(t_{m-1}\right), & u^{m, \nu}:=\nu u\left(t_{m}\right)+(1-\nu) u\left(t_{m-1}\right), \\
E_{u}^{m, \nu}:=\nu E_{u}^{m}+(1-\nu) E_{u}^{m-1}, & E_{\varphi}^{m, \mu}:=\mu E_{\varphi}^{m}+(1-\mu) E_{\varphi}^{m-1} .
\end{array}
$$

Since the proof is long and technical, we divide it into four steps.

Step 1. Test (1.5) by $v_{h} \in V^{h}$ and (1.6) by $\psi_{h} \in V^{h}$, respectively, and subtract the resulting equations from (3.1) and (3.2), respectively yield the following error equations:

$$
\begin{array}{r}
\left(d_{t} E_{u}^{m}, v_{h}\right)+\alpha k_{\varepsilon}\left(\nabla E_{u}^{m}, \nabla v_{h}\right)+\alpha\left(\left|\varphi\left(t_{m}\right)\right|^{2} \nabla u\left(t_{m}\right)-\left|\varphi_{h}^{m, \mu}\right|^{2} \nabla u_{h}^{m}, \nabla v_{h}\right)+\left(\gamma E_{u}^{m}, v_{h}\right)=\left(\mathcal{R}_{u}^{m}, v_{h}\right) \\
\forall v_{h} \in V^{h} \\
\left(d_{t} E_{\varphi}^{m}, \psi_{h}\right)+2 \beta \varepsilon\left(\nabla E_{\varphi}^{m}, \nabla \psi_{h}\right)+\alpha\left(\left|\nabla u\left(t_{m}\right)\right|^{2} \varphi\left(t_{m}\right)-\left|\nabla u_{h}^{m, \nu}\right|^{2} \varphi_{h}^{m}, \psi_{h}\right)+\frac{\beta}{2 \varepsilon}\left(E_{\varphi}^{m}, \psi_{h}\right)=\left(\mathcal{R}_{\varphi}^{m}, \psi_{h}\right) \\
\forall \psi_{h} \in V^{h},
\end{array}
$$

where

$$
\mathcal{R}_{u}^{m}:=u_{t}\left(t_{m}\right)-d_{t} u\left(t_{m}\right), \quad \mathcal{R}_{\varphi}^{m}:=\varphi_{t}\left(t_{m}\right)-d_{t} \varphi\left(t_{m}\right)
$$

Let

$$
\Theta_{u}^{m}:=u\left(t_{m}\right)-P_{h} u\left(t_{m}\right), \quad \Theta_{\varphi}^{m}:=\varphi\left(t_{m}\right)-P_{h} \varphi\left(t_{m}\right)
$$

Taking

$$
v_{h}=P_{h} u\left(t_{m}\right)-u_{h}^{m}=E_{u}^{m}-\Theta_{u}^{m} \quad \text { and } \quad \psi_{h}=P_{h} \varphi\left(t_{m}\right)-\varphi_{h}^{m}=E_{\varphi}^{m}-\Theta_{\varphi}^{m}
$$


in (3.59) and (3.60), respectively, we have

$$
\begin{gathered}
\frac{1}{2} d_{t}\left\|E_{u}^{m}\right\|_{L^{2}}^{2}+\frac{k}{2}\left\|d_{t} E_{u}^{m}\right\|_{L^{2}}^{2}+\alpha k_{\varepsilon}\left\|\nabla E_{u}^{m}\right\|_{L^{2}}^{2}+\left\|\sqrt{\gamma} E_{u}^{m}\right\|_{L^{2}}^{2}+\alpha\left(\left|\varphi\left(t_{m}\right)\right|^{2} \nabla u\left(t_{m}\right)-\left|\varphi_{h}^{m, \mu}\right|^{2} \nabla u_{h}^{m}, \nabla E_{u}^{m}\right) \\
=\left(\mathcal{R}_{u}^{m}, \Theta_{u}^{m}-E_{u}^{m}\right)+\left(d_{t} E_{u}^{m}, \Theta_{u}^{m}\right)+\alpha k_{\varepsilon}\left(\nabla E_{u}^{m}, \nabla \Theta_{u}^{m}\right)+\left(\gamma E_{u}^{m}, \Theta_{u}^{m}\right) \\
+\alpha\left(\left|\varphi\left(t_{m}\right)\right|^{2} \nabla u\left(t_{m}\right)-\left|\varphi_{h}^{m, \mu}\right|^{2} \nabla u_{h}^{m}, \nabla \Theta_{u}^{m}\right) \\
\frac{1}{2} d_{t}\left\|E_{\varphi}^{m}\right\|_{L^{2}}^{2}+\frac{k}{2}\left\|d_{t} E_{\varphi}^{m}\right\|_{L^{2}}^{2}+2 \beta \varepsilon\left\|\nabla E_{\varphi}^{m}\right\|_{L^{2}}^{2}+\frac{\beta}{2 \varepsilon}\left\|E_{\varphi}^{m}\right\|_{h}^{2}+\alpha\left(\left|\nabla u\left(t_{m}\right)\right|^{2} \varphi\left(t_{m}\right)-\left|\nabla u_{h}^{m, \nu}\right|^{2} \varphi_{h}^{m}, E_{\varphi}^{m}\right) \\
=\left(\mathcal{R}_{\varphi}^{m}, \Theta_{\varphi}^{m}-E_{\varphi}^{m}\right)+\left(d_{t} E_{\varphi}^{m}, \Theta_{\varphi}^{m}\right)+2 \beta \varepsilon\left(\nabla E_{\varphi}^{m}, \nabla \Theta_{\varphi}^{m}\right)+\frac{\beta}{2 \varepsilon}\left(E_{\varphi}^{m}, \Theta_{\varphi}^{m}\right) \\
+\alpha\left(\left|\nabla u\left(t_{m}\right)\right|^{2} \varphi\left(t_{m}\right)-\left|\nabla u_{h}^{m, \nu}\right|^{2} \varphi_{h}^{m}, \Theta_{\varphi}^{m}\right) .
\end{gathered}
$$

Step 2. It remains to bound all inner product terms in (3.61) and (3.62). Clearly, each linear term can be easily bounded from above using standard techniques. On the other hand, bounding four nonlinear terms is more complicated, they need to be handled carefully in order to obtain the desired error estimate.

Using the algebraic relations

$$
\begin{aligned}
& \varphi\left(t_{m}\right)-\varphi_{h}^{m, \mu}=(1-\mu) k d_{t} \varphi\left(t_{m}\right)+E_{\varphi}^{m, \mu}, \\
& \varphi\left(t_{m}\right)+\varphi_{h}^{m, \mu}=2 \varphi\left(t_{m}\right)-(1-\mu) k d_{t} \varphi\left(t_{m}\right)-E_{\varphi}^{m, \mu},
\end{aligned}
$$

we have

$$
\begin{aligned}
\mathcal{A}_{1}:= & \left|\varphi\left(t_{m}\right)\right|^{2} \nabla u\left(t_{m}\right)-\left|\varphi_{h}^{m, \mu}\right|^{2} \nabla u_{h}^{m} \\
= & \left|\varphi\left(t_{m}\right)\right|^{2} \nabla E_{u}^{m}+\left[\varphi\left(t_{m}\right)-\varphi_{h}^{m, \mu}\right]\left[\varphi\left(t_{m}\right)+\varphi_{h}^{m, \mu}\right] \nabla u_{h}^{m} \\
= & \left|\varphi\left(t_{m}\right)\right|^{2} \nabla E_{u}^{m}+\left[2(1-\mu) k d_{t} \varphi\left(t_{m}\right) \varphi\left(t_{m}\right)-(1-\mu)^{2} k^{2}\left|d_{t} \varphi\left(t_{m}\right)\right|^{2}\right. \\
& \left.-2(1-\mu) k d_{t} \varphi\left(t_{m}\right) E_{\varphi}^{m, \mu}+2 \varphi\left(t_{m}\right) E_{\varphi}^{m, \mu}-\left|E_{\varphi}^{m, \mu}\right|^{2}\right]\left[\nabla u\left(t_{m}\right)-\nabla E_{u}^{m}\right] .
\end{aligned}
$$

Then, it follows from Schwarz inequality and the fact that $0 \leq \varphi\left(t_{m}\right) \leq 1$

$$
\begin{aligned}
\left(\mathcal{A}_{1}, \nabla E_{u}^{m}\right) \geq & \left\|\varphi\left(t_{m}\right) \nabla E_{u}^{m}\right\|_{L^{2}}^{2}-\left\|\nabla u\left(t_{m}\right)\right\|_{L^{\infty}}\left\{2(1-\mu) k\left\|d_{t} \varphi\left(t_{m}\right)\right\|_{L^{2}}\left\|\varphi\left(t_{m}\right) \nabla E_{u}^{m}\right\|_{L^{2}}\right. \\
& +(1-\mu)^{2} k^{2}\left\|d_{t} \varphi\left(t_{m}\right)\right\|_{L^{4}}^{2}\left\|\nabla E_{u}^{m}\right\|_{L^{2}}+2(1-\mu) k\left\|d_{t} \varphi\left(t_{m}\right)\right\|_{L^{2}}\left\|E_{\varphi}^{m, \mu} \nabla E_{u}^{m}\right\|_{L^{2}} \\
& \left.+2\left\|\varphi\left(t_{m}\right) \nabla E_{u}^{m}\right\|_{L^{2}}\left\|E_{\varphi}^{m, \mu}\right\|_{L^{2}}+\left\|E_{\varphi}^{m, \mu} \nabla E_{u}^{m}\right\|_{L^{2}}\left\|E_{\varphi}^{m, \mu}\right\|_{L^{2}}\right\} \\
& -\left\|\nabla E_{u}^{m}\right\|_{L^{2}}^{2}\left\{2(1-\mu) k\left\|d_{t} \varphi\left(t_{m}\right)\right\|_{L^{\infty}}+(1-\mu)^{2} k^{2}\left\|d_{t} \varphi\left(t_{m}\right)\right\|_{L^{\infty}}^{2}\right\} \\
& -2(1-\mu) k\left\|d_{t} \varphi\left(t_{m}\right)\right\|_{L^{\infty}}\left\|E_{\varphi}^{m, \mu} \nabla E_{u}^{m}\right\|_{L^{2}}\left\|\nabla E_{u}^{m}\right\|_{L^{2}} \\
& -2\left\|E_{\varphi}^{m, \mu} \nabla E_{u}^{m}\right\|_{L^{2}}\left\|\varphi\left(t_{m}\right) \nabla E_{u}^{m}\right\|_{L^{2}}+\left\|E_{\varphi}^{m, \mu} \nabla E_{u}^{m}\right\|_{L^{2}}^{2} \\
\geq & \frac{1+\mu}{4}\left\|\varphi\left(t_{m}\right) \nabla E_{u}^{m}\right\|_{L^{2}}^{2}-\left[\frac{(1-\mu)^{2} k_{\varepsilon}}{4}+2(1-\mu) k\left\|d_{t} \varphi\left(t_{m}\right)\right\|_{L^{\infty}}\right. \\
& \left.+2(1-\mu)^{2} k^{2}\left\|d_{t} \varphi\left(t_{m}\right)\right\|_{L^{\infty}}^{2}\right]\left\|\nabla E_{u}^{m}\right\|_{L^{2}}^{2}-5\left\|\nabla u\left(t_{m}\right)\right\|_{L^{\infty}}^{2}\left\|E_{\varphi}^{m, \mu}\right\|_{L^{2}}^{2} \\
& -(5+\mu)\left\|E_{\varphi}^{m, \mu} \nabla E_{u}^{m}\right\|_{L^{2}}^{2}-k^{2}\left[5(1-\mu)\left\|d_{t} \varphi\left(t_{m}\right)\right\|_{L^{2}}^{2}\right. \\
& \left.+\frac{(1-\mu)^{2} k^{2}}{k_{\varepsilon}}\left\|d_{t} \varphi\left(t_{m}\right)\right\|_{L^{4}}^{4}\right]\left\|\nabla u\left(t_{m}\right)\right\|_{L^{\infty}}^{2}
\end{aligned}
$$


and

$$
\begin{gathered}
\left|\left(\mathcal{A}_{1}, \nabla \Theta_{u}^{m}\right)\right| \leq \frac{1}{16}\left\|\varphi\left(t_{m}\right) \nabla E_{u}^{m}\right\|_{L^{2}}^{2}+\left[3+\frac{1}{\varepsilon}\left\|\nabla u\left(t_{m}\right)\right\|_{L^{\infty}}^{2}\left\|\nabla \Theta_{u}^{m}\right\|_{L^{2}}^{2}\right]\left\|E_{\varphi}^{m, \mu}\right\|_{L^{2}}^{2} \\
+\frac{\varepsilon}{16}\left\|\nabla E_{\varphi}^{m, \mu}\right\|_{L^{2}}^{2}+4(1-\mu)^{4} k^{4}\left\|d_{t} \varphi\left(t_{m}\right)\right\|_{L^{\infty}}^{4}\left\|\nabla E_{u}^{m}\right\|_{L^{2}}^{2} \\
+2\left[1+\left\|\nabla \Theta_{u}^{m}\right\|_{L^{\infty}}^{2}\right]\left\|E_{\varphi}^{m, \mu} \nabla E_{u}^{m}\right\|_{L^{2}}^{2}+12\left\|\nabla \Theta_{u}^{m}\right\|_{L^{2}}^{2}+(1-\mu) k^{2}\left\|d_{t} \varphi\left(t_{m}\right)\right\|_{L^{2}}^{2} .
\end{gathered}
$$

Similarly, using the algebraic relations

$$
\begin{aligned}
\nabla u\left(t_{m}\right)-\nabla u_{h}^{m, \nu} & =(1-\nu) k d_{t} \nabla u\left(t_{m}\right)+E_{u}^{m, \nu} \\
\nabla u_{h}^{m, \nu} & =\nabla u\left(t_{m}\right)-(1-\nu) k d_{t} \nabla u\left(t_{m}\right)-\nabla E_{u}^{m, \nu} \\
\varphi_{h}^{m} & =\varphi\left(t_{m}\right)-E_{\varphi}^{m}
\end{aligned}
$$

we have

$$
\begin{aligned}
\mathcal{A}_{2} & :=\left|\nabla u\left(t_{m}\right)\right|^{2} \varphi\left(t_{m}\right)-\left|\nabla u_{h}^{m, \nu}\right|^{2} \varphi_{h}^{m} \\
& =\left|\nabla u\left(t_{m}\right)\right|^{2} E_{\varphi}^{m}+\left(\nabla u\left(t_{m}\right)-\nabla u_{h}^{m, \nu}, \nabla u\left(t_{m}\right)+\nabla u_{h}^{m, \nu}\right) \varphi_{h}^{m} \\
& =\left|\nabla u\left(t_{m}\right)\right|^{2} E_{\varphi}^{m}+\left((1-\nu) k d_{t} \nabla u\left(t_{m}\right)+E_{u}^{m, \nu}, 2 \nabla u\left(t_{m}\right)-(1-\nu) k d_{t} \nabla u\left(t_{m}\right)-\nabla E_{u}^{m, \nu}\right)\left(\varphi\left(t_{m}\right)-E_{\varphi}^{m}\right) .
\end{aligned}
$$

Then

$$
\begin{aligned}
\left(\mathcal{A}_{2}, E_{\varphi}^{m}\right) \geq & \left\|\nabla u\left(t_{m}\right) E_{\varphi}^{m}\right\|_{L^{2}}^{2}-(1-\nu) k\left\|d_{t} \nabla u\left(t_{m}\right)\right\|_{L^{2}}\left\{2\left\|\nabla u\left(t_{m}\right) E_{\varphi}^{m}\right\|_{L^{2}}\right. \\
& \left.+(1-\nu) k\left\|d_{t} \nabla u\left(t_{m}\right)\right\|_{L^{\infty}}\left\|E_{\varphi}^{m}\right\|_{L^{2}}+2\left\|E_{\varphi}^{m} \nabla E_{u}^{m, \nu}\right\|_{L^{2}}\right\} \\
& -\left\|E_{\varphi}^{m} \nabla E_{u}^{m, \nu}\right\|_{L^{2}}\left\{(1-\nu) k\left\|d_{t} \nabla u\left(t_{m}\right)\right\|_{L^{2}}+\left\|\varphi\left(t_{m}\right) \nabla E_{u}^{m, \nu}\right\|_{L^{2}}\right\} \\
& -2(1-\nu) k\left\|d_{t} \nabla u\left(t_{m}\right)\right\|_{L^{\infty}}\left\|E_{\varphi}^{m}\right\|_{L^{2}}\left\{\left\|\nabla u\left(t_{m}\right) E_{\varphi}^{m}\right\|_{L^{2}}+\left\|E_{\varphi}^{m} \nabla E_{u}^{m, \nu}\right\|_{L^{2}}\right\} \\
& +(1-\nu)^{2} k^{2}\left\|d_{t} \nabla u\left(t_{m}\right) E_{\varphi}^{m}\right\|_{L^{2}}^{2}+\left\|E_{\varphi}^{m} \nabla E_{u}^{m, \nu}\right\|_{L^{2}}^{2} \\
\geq & \frac{1}{2}\left\|\nabla u\left(t_{m}\right) E_{\varphi}^{m}\right\|_{L^{2}}^{2}-\frac{1}{16}\left\|\varphi\left(t_{m}\right) \nabla E_{u}^{m, \nu}\right\|_{L^{2}}^{2}-12\left\|E_{\varphi}^{m} \nabla E_{u}^{m, \nu}\right\|_{L^{2}}^{2} \\
& -4\left\{(1-\nu) k\left\|d_{t} \nabla u\left(t_{m}\right)\right\|_{L^{\infty}}^{2}+\left\|\nabla u\left(t_{m}\right)\right\|_{L^{\infty}}^{2}\right\}\left\|E_{\varphi}^{m}\right\|_{L^{2}}^{2}-4(1-\nu)^{2} k^{2}\left\|d_{t} \nabla u\left(t_{m}\right)\right\|_{L^{2}}^{2},
\end{aligned}
$$

and

$$
\begin{aligned}
\left|\left(\mathcal{A}_{2}, \Theta_{\varphi}^{m}\right)\right| \leq & \frac{1}{4}\left\|\nabla u\left(t_{m}\right) E_{\varphi}^{m}\right\|_{L^{2}}^{2}+\frac{1}{16}\left\|\varphi\left(t_{m}\right) \nabla E_{u}^{m, \nu}\right\|_{L^{2}}^{2}+8\left\|\Theta_{\varphi}^{m}\right\|_{L^{\infty}}^{2}\left[\left\|E_{\varphi}^{m}\right\|_{L^{2}}^{2}+\left\|\nabla E_{u}^{m, \nu}\right\|_{L^{2}}^{2}\right] \\
& +8\left[1+\left\|\nabla u\left(t_{m}\right)\right\|_{L^{\infty}}^{2}\right]\left\|\Theta_{\varphi}^{m}\right\|_{L^{2}}^{2}+(1-\nu)^{4} k^{4}\left\|d_{t} \nabla u\left(t_{m}\right)\right\|_{L^{4}}^{4} \\
& +8(1-\nu)^{2} k^{2}\left[1+\left\|\Theta_{\varphi}^{m}\right\|_{L^{\infty}}^{2}\right]\left\|d_{t} \nabla u\left(t_{m}\right)\right\|_{L^{2}}^{2} .
\end{aligned}
$$


Next, we need to bound the factor $k\left\|d_{t} \varphi\left(t_{m}\right)\right\|_{L^{\infty}}$ which appears in (3.63)-(3.65). Using a Nirenberg-Gagliardo inequality [1] we have

$$
\begin{aligned}
k\left\|d_{t} \varphi\left(t_{m}\right)\right\|_{L^{\infty}} & \leq k\left[\left\|d_{t} \varphi\left(t_{m}\right)\right\|_{L^{2}}^{\frac{1}{2}}\left\|\nabla d_{t} \varphi\left(t_{m}\right)\right\|_{L^{\infty}}^{\frac{1}{2}}+\left\|d_{t} \varphi\left(t_{m}\right)\right\|_{L^{2}}\right] \\
& \leq k^{\frac{1}{2}}\left\|d_{t} \varphi\left(t_{m}\right)\right\|_{L^{2}}^{\frac{1}{2}}\left\|\nabla \varphi\left(t_{m}\right)-\nabla \varphi\left(t_{m-1}\right)\right\|_{L^{\infty}}^{\frac{1}{2}}+k\left\|d_{t} \varphi\left(t_{m}\right)\right\|_{L^{2}}, \\
& \leq 3 k^{\frac{1}{2}}\|\nabla \varphi\|_{L^{\infty}\left(\Omega_{T}\right)}^{\frac{1}{2}} .
\end{aligned}
$$

Finally, using the relations

$$
\begin{aligned}
& \left(d_{t} E_{u}^{m}, \Theta_{u}^{m}\right)=d_{t}\left(E_{u}^{m}, \Theta_{u}^{m}\right)-\left(E_{u}^{m-1}, d_{t} \Theta_{u}^{m}\right), \\
& \left(d_{t} E_{\varphi}^{m}, \Theta_{\varphi}^{m}\right)=d_{t}\left(E_{\varphi}^{m}, \Theta_{\varphi}^{m}\right)-\left(E_{\varphi}^{m-1}, d_{t} \Theta_{\varphi}^{m}\right),
\end{aligned}
$$

we can bound the linear terms in (3.61) and (3.62) follows:

$$
\begin{aligned}
\left|\left(\mathcal{R}_{u}^{m}, \Theta_{u}^{m}-E_{u}^{m}\right)-\left(E_{u}^{m-1}, d_{t} \Theta_{u}^{m}\right)+\alpha k_{\varepsilon}\left(\nabla E_{u}^{m}, \nabla \Theta_{u}^{m}\right)+\left(\gamma E_{u}^{m}, \Theta_{u}^{m}\right)\right| \leq \frac{\alpha k_{\varepsilon}}{4}\left[\left\|\nabla E_{u}^{m-1}\right\|_{L^{2}}^{2}+\left\|\nabla E_{u}^{m}\right\|_{L^{2}}^{2}\right] \\
+\frac{1}{2}\left\|\sqrt{\gamma} E_{u}^{m}\right\|_{L^{2}}^{2}+\frac{C}{\alpha k_{\varepsilon}}\left[\left\|\mathcal{R}_{u}^{m}\right\|_{H^{-1}}^{2}+\left\|d_{t} \Theta_{u}^{m}\right\|_{H^{-1}}^{2}\right]+C\left[\alpha k_{\varepsilon}\left\|\nabla \Theta_{u}^{m}\right\|_{L^{2}}^{2}+\left\|\sqrt{\gamma} \Theta_{u}^{m}\right\|_{L^{2}}^{2}\right], \quad \text { (3.68) } \\
\left|\left(\mathcal{R}_{\varphi}^{m}, \Theta_{\varphi}^{m}-E_{\varphi}^{m}\right)-\left(E_{\varphi}^{m-1}, d_{t} \Theta_{\varphi}^{m}\right)+2 \beta \varepsilon\left(\nabla E_{\varphi}^{m}, \nabla \Theta_{\varphi}^{m}\right)+\frac{\beta}{2 \varepsilon}\left(E_{\varphi}^{m}, \Theta_{\varphi}^{m}\right)\right| \leq \beta \varepsilon\left[\left\|\nabla E_{\varphi}^{m-1}\right\|_{L^{2}}^{2}+\left\|\nabla E_{\varphi}^{m}\right\|_{L^{2}}^{2}\right] \\
+\frac{\beta}{4 \varepsilon}\left\|E_{\varphi}^{m}\right\|_{L^{2}}^{2}+\frac{C}{\beta \varepsilon}\left[\left\|\mathcal{R}_{\varphi}^{m}\right\|_{H^{-1}}^{2}+\left\|d_{t} \Theta_{\varphi}^{m}\right\|_{H^{-1}}^{2}\right]+C\left[\beta \varepsilon\left\|\nabla \Theta_{\varphi}^{m}\right\|_{L^{2}}^{2}+\frac{\beta}{4 \varepsilon}\left\|\Theta_{\varphi}^{m}\right\|_{L^{2}}^{2}\right] .
\end{aligned}
$$

Since $\mathcal{R}_{u}^{m}$ and $\mathcal{R}_{\varphi}^{m}$ can be written as

$$
\mathcal{R}_{u}^{m}=\frac{1}{k} \int_{t_{m}}^{t_{m+1}}\left(s-t_{m}\right) u_{t t}(s) \mathrm{d} s, \quad \mathcal{R}_{\varphi}^{m}=\frac{1}{k} \int_{t_{m}}^{t_{m+1}}\left(s-t_{m}\right) \varphi_{t t}(s) \mathrm{d} s,
$$

from Lemma 2.1 we have

$$
\begin{aligned}
& k \sum_{m=0}^{M}\left\|\mathcal{R}_{u}^{m}\right\|_{H^{-1}}^{2} \leq k \sum_{m=0}^{M}\left[\int_{t_{m}}^{t_{m+1}}\left(s-t_{m}\right)^{2} \mathrm{~d} s\right]\left[\int_{t_{m}}^{t_{m+1}}\left\|u_{t t}(s)\right\|_{H^{-1}}^{2} \mathrm{~d} s\right] \leq C_{3} k^{2}, \\
& k \sum_{m=0}^{M}\left\|\mathcal{R}_{\varphi}^{m}\right\|_{H^{-1}}^{2} \leq k \sum_{m=0}^{M}\left[\int_{t_{m}}^{t_{m+1}}\left(s-t_{m}\right)^{2} \mathrm{~d} s\right]\left[\int_{t_{m}}^{t_{m+1}}\left\|\varphi_{t t}(s)\right\|_{H^{-1}}^{2} \mathrm{~d} s\right] \leq C_{3} k^{2} .
\end{aligned}
$$


Step 3. Now, substituting the estimates (3.63)-(3.71) into (3.61) and (3.62), respectively, adding the resulted inequalities and applying the operator $k \sum_{k=1}^{\ell}(\ell \leq M)$ we get

$$
\begin{aligned}
& \left\|E_{u}^{\ell}\right\|_{L^{2}}^{2}+\left\|E_{\varphi}^{\ell}\right\|_{L^{2}}^{2}+k \sum_{m=1}^{\ell}\left\{k\left\|d_{t} E_{u}^{m}\right\|_{L^{2}}^{2}+k\left\|d_{t} E_{\varphi}^{m}\right\|_{L^{2}}^{2}\right. \\
& +\left[\alpha k_{\varepsilon}-c_{0}(1-\mu) k\|\nabla \varphi\|_{L^{\infty}\left(L^{\infty}\right)}-c_{0}(1-\nu)\left\|\Theta_{\varphi}\right\|_{L^{\infty}\left(L^{\infty}\right)}^{2}\right]\left\|\nabla E_{u}^{m}\right\|_{L^{2}}^{2} \\
& +\alpha\left\|\varphi\left(t_{m}\right) \nabla E_{u}^{m}\right\|_{L^{2}}^{2}+\left\|\sqrt{\gamma} E_{u}^{m}\right\|_{L^{2}}^{2}+\beta \varepsilon\left\|\nabla E_{\varphi}^{m}\right\|_{L^{2}}^{2}+\alpha\left\|\nabla u\left(t_{m}\right) E_{\varphi}^{m}\right\|_{L^{2}}^{2} \\
& \left.+\left[\frac{\beta}{\varepsilon}-c_{0}\|\nabla u\|_{L^{\infty}\left(L^{\infty}\right)}^{2}-\frac{c_{0}}{\varepsilon}\|\nabla u\|_{L^{\infty}\left(L^{\infty}\right)}^{2}\left\|\nabla \Theta_{u}\right\|_{L^{\infty}\left(L^{2}\right)}^{2}-c_{0}\left\|\Theta_{\varphi}\right\|_{L^{\infty}\left(L^{\infty}\right)}^{2}\right]\left\|E_{\varphi}^{m}\right\|_{L^{2}}^{2}\right\} \\
& \leq C k \sum_{m=1}^{\ell}\left\{\left\|E_{u}^{m}\right\|_{L^{2}}^{2}+\left\|E_{\varphi}^{m, \mu}\right\|_{L^{2}}^{2}+\left(1+\left\|\nabla \Theta_{u}^{m}\right\|_{L^{\infty}}^{2}\right)\left\|E_{\varphi}^{m, \mu} \nabla E_{u}^{m}\right\|_{L^{2}}^{2}+\left\|E_{\varphi}^{m} \nabla E_{u}^{m, \nu}\right\|_{L^{2}}^{2}\right\} \\
& +\frac{k}{4} \sum_{m=1}^{\ell}\left\{(1-\mu)\left\|\nabla u\left(t_{m}\right)\right\|_{L^{\infty}}^{2}\left\|E_{\varphi}^{m, \mu}\right\|_{L^{2}}^{2}+\alpha(1-\nu)\left\|\varphi\left(t_{m}\right) \nabla E_{u}^{m, \nu}\right\|_{L^{2}}^{2}+\beta \varepsilon(1-\mu)\left\|\nabla E_{\varphi}^{m, \mu}\right\|_{L^{2}}^{2}\right\} \\
& +C k^{2}(1-\nu) k \sum_{m=1}^{\ell}\left\|\Theta_{\varphi}^{m}\right\|_{L^{\infty}}^{2}\left\|d_{t} \nabla u\left(t_{m}\right)\right\|_{L^{2}}^{2}+C k^{2}(1-\mu) k \sum_{m=1}^{\ell}\left\|\nabla u\left(t_{m}\right)\right\|_{L^{\infty}}^{2}\left\|d_{t} \varphi\left(t_{m}\right)\right\|_{L^{2}}^{2} \\
& +C k^{5} \sum_{m=1}^{\ell}\left\{\frac{1-\mu}{k_{\varepsilon}}\left\|\nabla u\left(t_{m}\right)\right\|_{L^{\infty}}^{2}\left\|d_{t} \varphi\left(t_{m}\right)\right\|_{L^{4}}^{4}+(1-\nu)\left\|d_{t} \nabla u\left(t_{m}\right)\right\|_{L^{4}}^{4}\right\} \\
& +C k \sum_{m=1}^{\ell}\left[\left\|\nabla \Theta_{u}^{m}\right\|_{L^{2}}^{2}+\|\nabla u\|_{L^{\infty}\left(L^{\infty}\right)}^{2}\left\|\Theta_{\varphi}^{m}\right\|_{L^{2}}^{2}\right] \\
& +C k \sum_{m=1}^{\ell}\left\{\frac{1}{\alpha k_{\varepsilon}}\left\|d_{t} \Theta_{u}^{m}\right\|_{H^{-1}}^{2}+\frac{1}{\beta \varepsilon}\left\|d_{t} \Theta_{\varphi}^{m}\right\|_{H^{-1}}^{2}+\alpha k_{\varepsilon}\left\|\nabla \Theta_{u}^{m}\right\|_{L^{2}}^{2}\right. \\
& \left.+\beta \varepsilon\left\|\nabla \Theta_{\varphi}^{m}\right\|_{L^{2}}^{2}+\frac{\beta}{4 \varepsilon}\left\|\Theta_{\varphi}^{m}\right\|_{L^{2}}^{2}\right\}+\left\|E_{u}^{0}\right\|_{L^{2}}^{2}+\left\|E_{\varphi}^{0}\right\|_{L^{2}}^{2}
\end{aligned}
$$

for some positive constant $c_{0}$ which is independent of $\varepsilon, k_{\varepsilon}, u$ and $\varphi$.

In view of (3.53) and the mesh condition (3.56), we have

$$
c^{*}:=\alpha k_{\varepsilon}-c_{0}(1-\mu) k\|\nabla \varphi\|_{L^{\infty}\left(L^{\infty}\right)}-c_{0}(1-\nu)\left\|\Theta_{\varphi}\right\|_{L^{\infty}\left(L^{\infty}\right)}^{2}>0 .
$$

Also, the assumption (3.54) and the mesh condition (3.56) imply that

$$
\frac{\beta}{\varepsilon}-c_{0}\|\nabla u\|_{L^{\infty}\left(L^{\infty}\right)}^{2}-\frac{c_{0}}{\varepsilon}\|\nabla u\|_{L^{\infty}\left(L^{\infty}\right)}^{2}\left\|\nabla \Theta_{u}\right\|_{L^{\infty}\left(L^{2}\right)}^{2}-c_{0}\left\|\Theta_{\varphi}\right\|_{L^{\infty}\left(L^{\infty}\right)}^{2}>0
$$

Hence, all terms (in the sum) on the left-hand side of (3.72) are positive. We also remark that when $\mu \neq 1$ and $\nu \neq 1$, the second sum on the right-hand side of (3.72) can be absorbed by the sum on the left-hand side.

Step 4. We now conclude the proof using a fixed point argumentation. Suppose that

$$
\max _{0 \leq m \leq M}\left[\left\|E_{u}^{m}\right\|_{L^{\infty}}^{2}+\left\|E_{\varphi}^{m}\right\|_{L^{\infty}}^{2}\right]<\frac{c^{*}}{4}
$$

then the third and fourth terms in the first sum on the right-hand side of (3.72) can be absorbed by the fifth term in the sum on the left-hand side. Applying the discrete Gronwall's inequality and Lemma 3.3 immediately 
yields the desired estimate (3.58). In particular, we have

$$
\max _{0 \leq m \leq M}\left[\left\|E_{u}^{m}\right\|_{L^{2}}+\left\|E_{\varphi}^{m}\right\|_{L^{2}}\right] \leq C(k+h),
$$

hence,

$$
\max _{0 \leq m \leq M}\left[\left\|P_{h} u\left(t_{m}\right)-u_{h}^{m}\right\|_{L^{2}}+\left\|P_{h} \varphi\left(t_{m}\right)-\varphi_{h}^{m}\right\|_{L^{2}}\right] \leq C(k+h) .
$$

By the inverse inequality bounding $L^{\infty}(\Omega)$ norm in terms of $L^{2}(\Omega)$ norm $[11,15]$, we get

$$
\begin{aligned}
\max _{0 \leq m \leq M}\left[\left\|P_{h} u\left(t_{m}\right)-u_{h}^{m}\right\|_{L^{\infty}}+\right. & \left.\left\|P_{h} \varphi\left(t_{m}\right)-\varphi_{h}^{m}\right\|_{L^{\infty}}\right] \leq \\
& C h^{-\frac{1}{2}} \max _{0 \leq m \leq M}\left[\left\|P_{h} u\left(t_{m}\right)-u_{h}^{m}\right\|_{L^{2}}+\left\|P_{h} \varphi\left(t_{m}\right)-\varphi_{h}^{m}\right\|_{L^{2}}\right] \leq C\left(k h^{-\frac{1}{2}}+h^{\frac{1}{2}}\right),
\end{aligned}
$$

which and (3.53) imply that

$$
\max _{0 \leq m \leq M}\left[\left\|E_{u}^{m}\right\|_{L^{\infty}}+\left\|E_{\varphi}^{m}\right\|_{L^{\infty}}\right] \leq C(\varepsilon)\left(h|\ln h|+k h^{-\frac{1}{2}}+h^{\frac{1}{2}}\right) .
$$

Clearly, (3.74) implies the induction assumption (3.73) for sufficient small $h$, and provided that $h, k$ satisfy the mesh relation $k=o\left(h^{\frac{1}{2}}\right)$. The proof is complete.

We conclude this section with a few remarks.

Remark 3.4. (a) The error estimates in (3.58) are optimal in $L^{2}\left((0, T) ; H^{1}(\Omega)\right)$-norm, and suboptimal in $L^{\infty}\left((0, T) ; L^{2}(\Omega)\right)$-norm. It might be possible to obtain optimal order error estimate of the form $O\left(k+h^{2}\right)$ in $L^{\infty}\left((0, T) ; L^{2}(\Omega)\right)$-norm by replacing the standard elliptic projection operator $P_{h}$ by some nonlinear projection operator $R_{h}$ associated with the underlying differential problem (1.5)-(1.8). If such an optimal order estimate can be obtained, it is easy to see that the conclusion of Theorem 3.3 also holds for $N=3$.

(b) The proof does not use the discrete stability estimates derived in Lemmas 3.1 and 3.2.

(c) As expected, the fully implicit scheme (i.e., $(\mu, \nu)=(1,1))$ produces a smaller error than its semi-implicit (i.e., $(\mu, \nu)=(0,1)$ and $(\mu, \nu)=(1,0))$ counterparts do, although they all have same asymptotic rate of convergence, since four terms on the right-hand side of $(3.72)$ vanish when $(\mu, \nu)=(1,1)$.

(d) Both $\left(u_{h}^{0}, \varphi_{h}^{0}\right)=\left(Q_{h} u_{0}, Q_{h} \varphi_{0}\right)$ and $\left(u_{h}^{0}, \varphi_{h}^{0}\right)=\left(I_{h} u_{0}, I_{h} \varphi_{0}\right)$ are valid choices of starting values for the schemes in (3.1)-(3.2) (cf. Rem. 3.3).

(e) The growth condition (3.54) is used to ensure positivity of the coefficient for $\left\|E_{\varphi}^{m}\right\|_{L^{2}}^{2}$ on the left-hand side of (3.72); as a result, the constant $\hat{C}$ in the error estimate (3.58) depends on $\frac{1}{\varepsilon}$ and $\frac{1}{k_{\varepsilon}}$ only in some low polynomial order. In Section 2, we have proved that this growth condition (3.54) holds at least locally in time. So a natural question is whether Theorem 3.3 still holds without assuming the growth condition (3.54). The answer to this question is yes. However, in this case since the coefficient for $\left\|E_{\varphi}^{m}\right\|_{L^{2}}^{2}$ on the left-hand side of (3.72) may not be positive, so we have to move this term to the right-hand side and treat it as a 'bad' term. As a result, the constant $\hat{C}$ in the error estimate (3.58) now depends on $\|\nabla u\|_{L^{\infty}\left(L^{\infty}\right)}^{2}$ exponentially, hence, it also depends on $\frac{1}{\varepsilon}$ and $\frac{1}{k_{\varepsilon}}$ exponentially. In this case, using a different interpolation of nonlinear terms that arise in the error analysis, it can be shown that the estimate also holds for $(u, \varphi) \in\left[L^{2}\left((0, T) ; H^{2}(\Omega)\right)\right]^{2}$.

\section{NUMERICAL EXPERIMENTS}

In this section we provide three sets of numerical experiments on the finite element methods developed in Section 3. Identical parameters $\alpha, \beta, \gamma, \varepsilon, h, k$ are used in the first two experiments, while the third experiment uses a larger $\gamma$, which is the coefficient in the fidelity term in the model. 

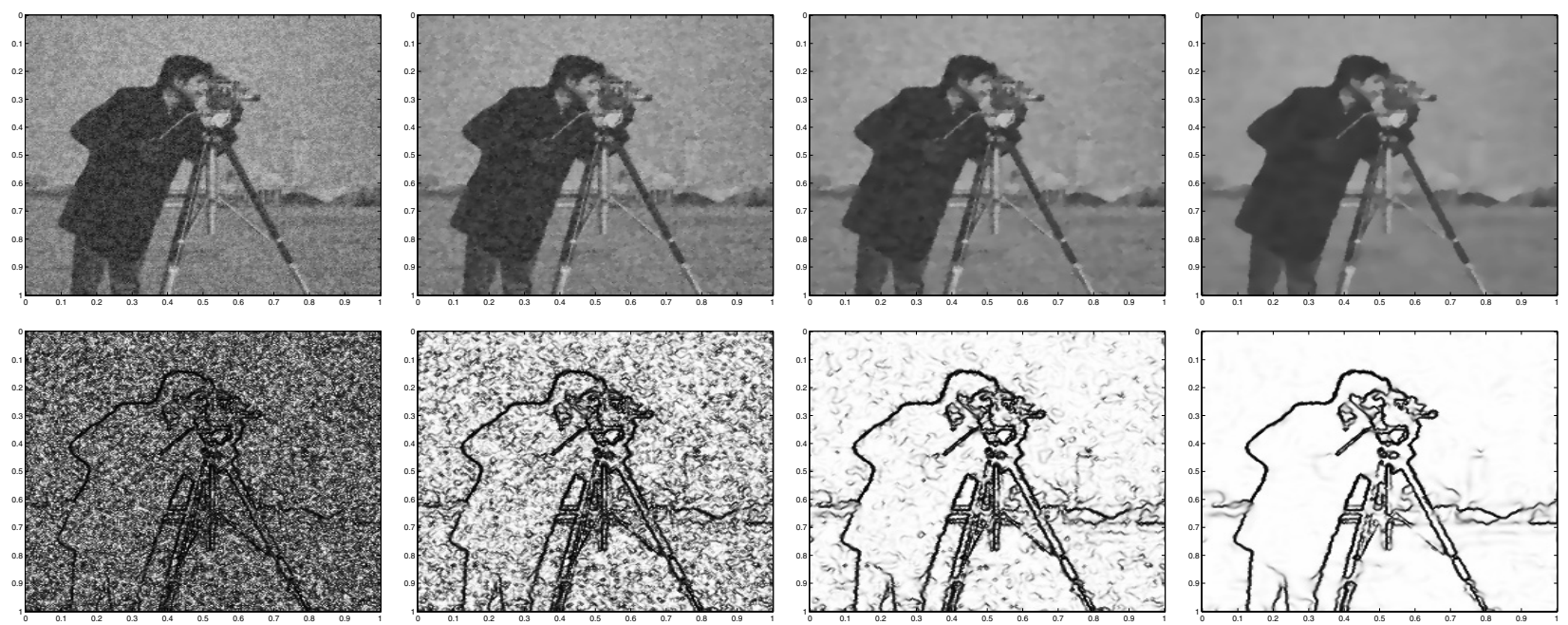

Figure 1. Evolution of $\left\{u_{h}^{m}\right\}$ (top row) and $\left\{\varphi_{h}^{m}\right\}$ (bottom row ) of Test 1.
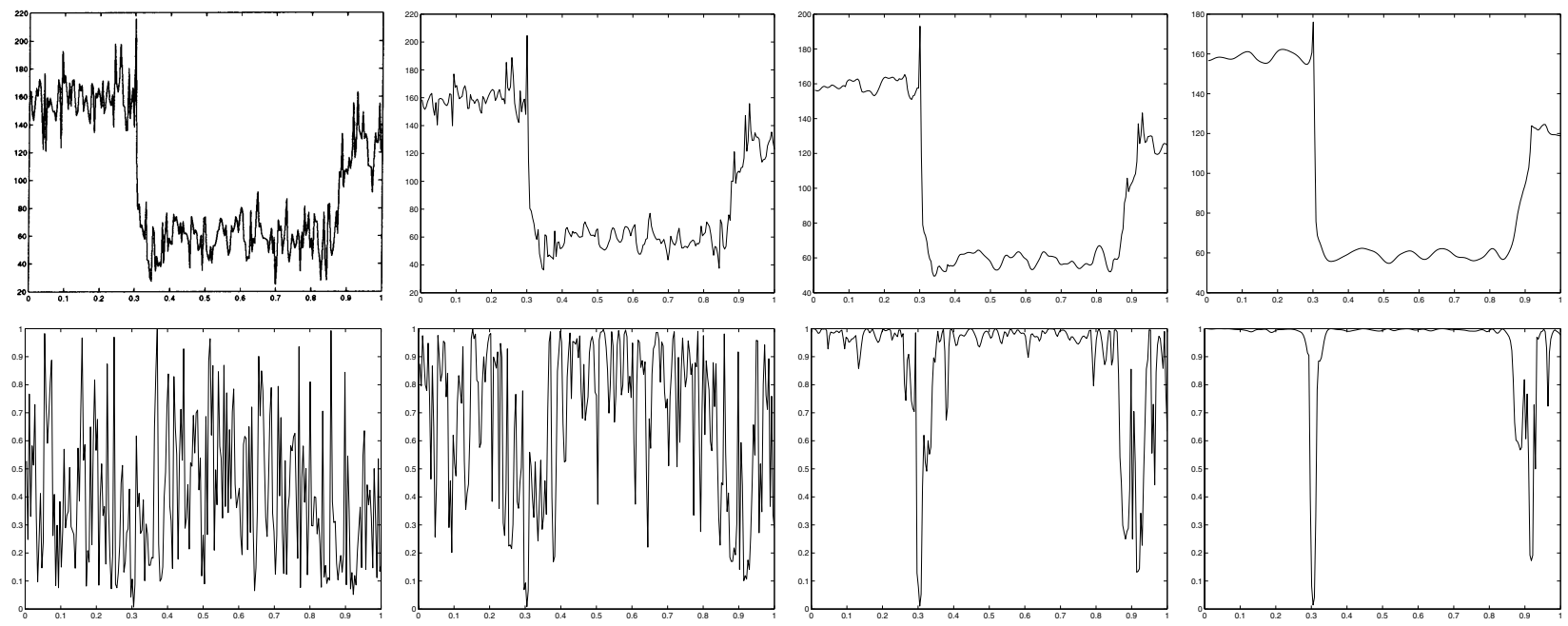

Figure 2. Cross sections at $x=60$ of the snapshots of Figure 1: $\left\{u_{h}^{m}\right\}$ (top row) and $\left\{\varphi_{h}^{m}\right\}$ (bottom row).

Test 1. The grid values of the initial function $u_{0}$ are chosen as a random perturbation of the grid values of the given image $g$ by a value between -90 and 90 , and the grid values of $\varphi_{0}$ are set to take the binary numbers 0 or 1 by a (boolean) thresholding criterion according to the corresponding grid values of $|\nabla g|$.

Starting with the above initial conditions, we compute the solution of the semi-implicit version of the scheme (3.1)-(3.2) (i.e., $\mu=\nu=0)$, with the physical parameters $(\alpha, \beta, \gamma)=\left(10^{-3}, 10^{-3}, 10^{-4}\right)$, the regularization parameters $\left(k_{\varepsilon}, \varepsilon\right)=\left(10^{-7}, 10^{-2}\right)$, and the numerical parameters $(h, k)=\left(4 \times 10^{-3}, 10^{-2}\right)$. We note that similar physical parameters $(\alpha, \beta, \gamma)$ were used in [28].

Figure 1 displays the computed soft image $u_{h}^{m}$ (top row) and edge function $\varphi_{h}^{m}$ (bottom row) at time steps $t_{1}, t_{4}, t_{8}$, and $t_{15}$. Figure 2 shows the cross section at $x=60$ of each $u_{h}^{m}$ and $\varphi_{h}^{m}$ of Figure 1 . Top rows of Figures 1 and 2 clearly show the denoising mechanism and the edge preserving property of the model and the numerical scheme. Also, the evolution of $\varphi_{h}^{m}$ shows a fast convergence to $\left|\varphi_{h}^{m}\right| \approx 1$ in the bulk, and to $\left|\varphi_{h}^{m}\right| \approx 0$ 

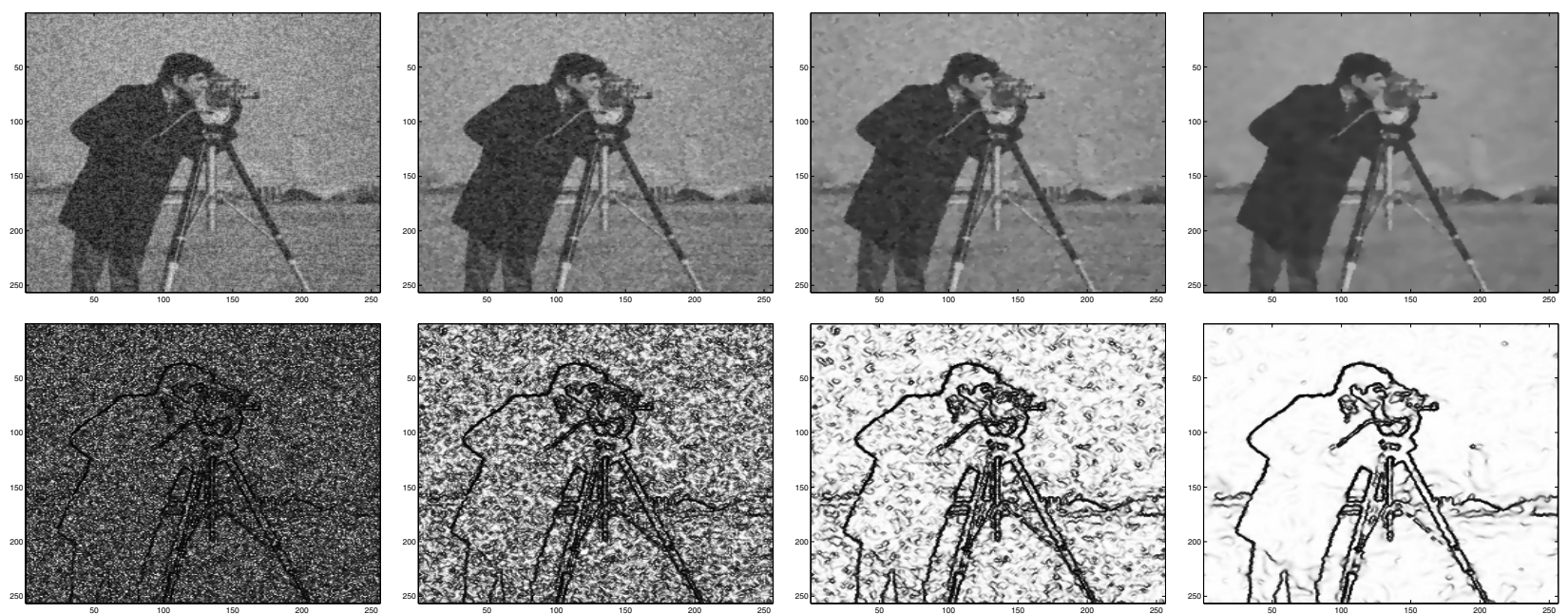

Figure 3. Evolution of $\left\{u_{h}^{m}\right\}$ (top row) and $\left\{\varphi_{h}^{m}\right\}$ (bottom row) of Test 2 .
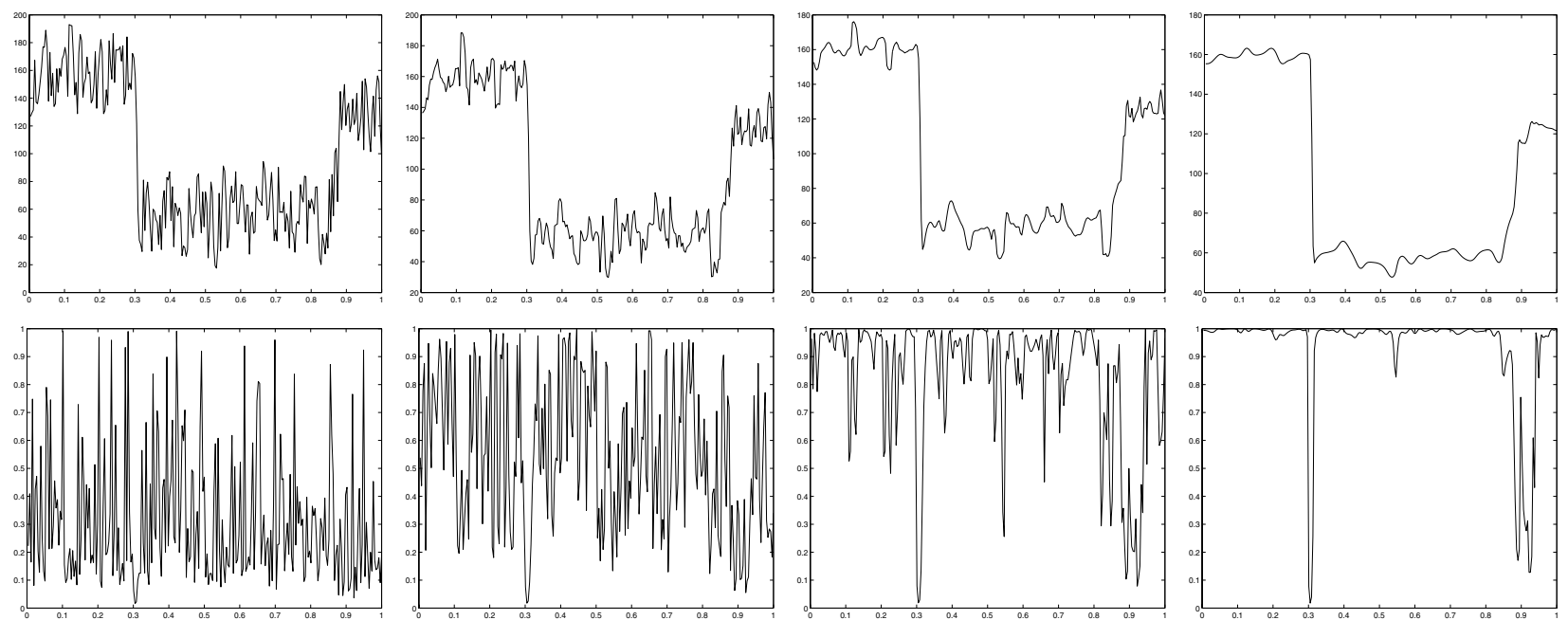

FiguRE 4. Cross sections at $x=60$ of the snapshots of Figure 3: $\left\{u_{h}^{m}\right\}$ (top row) and $\left\{\varphi_{h}^{m}\right\}$ (bottom row).

in a small region away from the bulk, which indicates the location of the edges. Oscillatory parts of $\varphi_{h}^{m}$ are quickly damped out when $t \rightarrow \infty$ as observed from the bottom rows of Figures 1 and 2 .

Test 2. Let $u_{0}$ be same as in Test 1 , set $\varphi_{0} \equiv 0.5$, and let all other parameters be same as in Test 1 .

Figure 3 displays the computed soft image $u_{h}^{m}$ (top row) and edge function $\varphi_{h}^{m}$ (bottom row) at time steps $t_{1}, t_{4}, t_{8}$, and $t_{15}$. Figure 4 shows the cross section at $x=60$ of each $u_{h}^{m}$ and $\varphi_{h}^{m}$ of Figure 3 . The same evolution as in Test 1 is observed. In addition, we observe $\varphi_{h}^{m}$ quickly and accurately detects edges. This indicates that the convergence of the gradient flow does not depend on the choice of the initial data $\varphi_{0}$, in particular, it does not have to reflect the edge information of the given image $g$. 

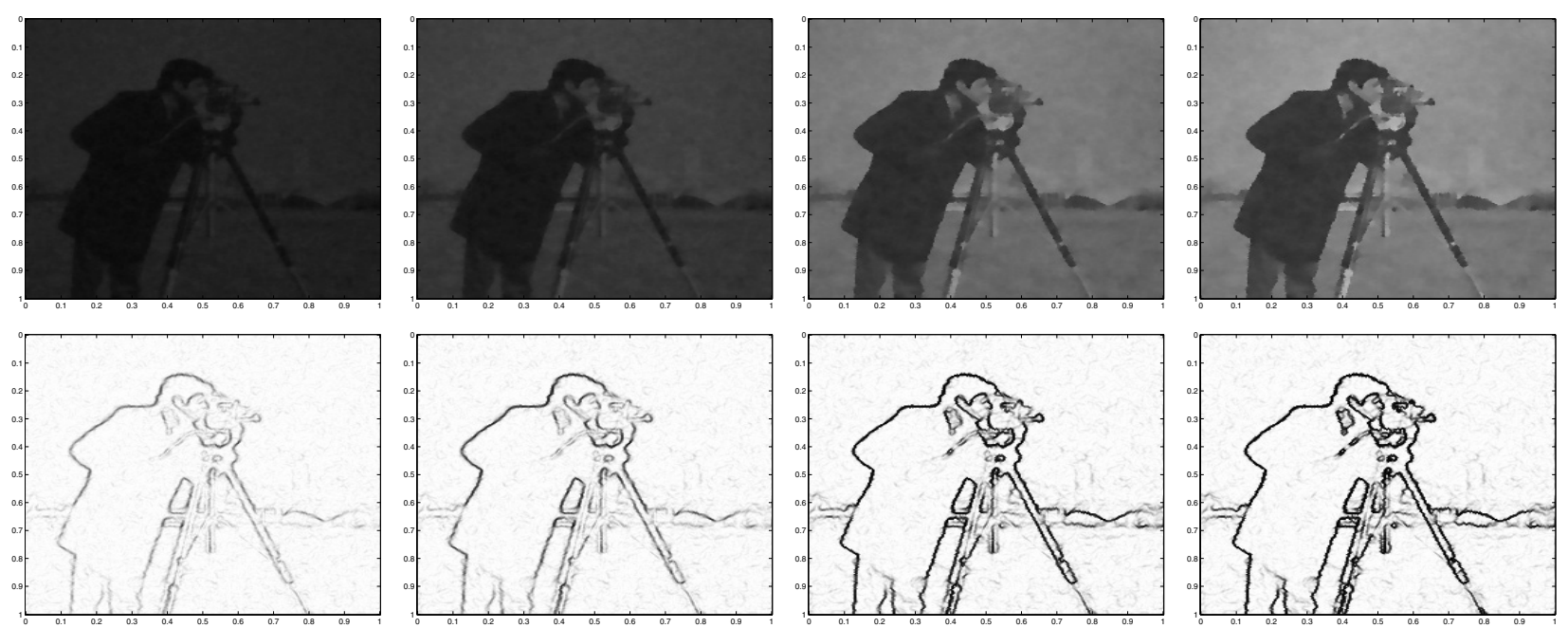

FiguRE 5. Evolution of $\left\{u_{h}^{m}\right\}$ (top row) and $\left\{v_{h}^{m}\right\}$ (bottom row) of Test 3 .
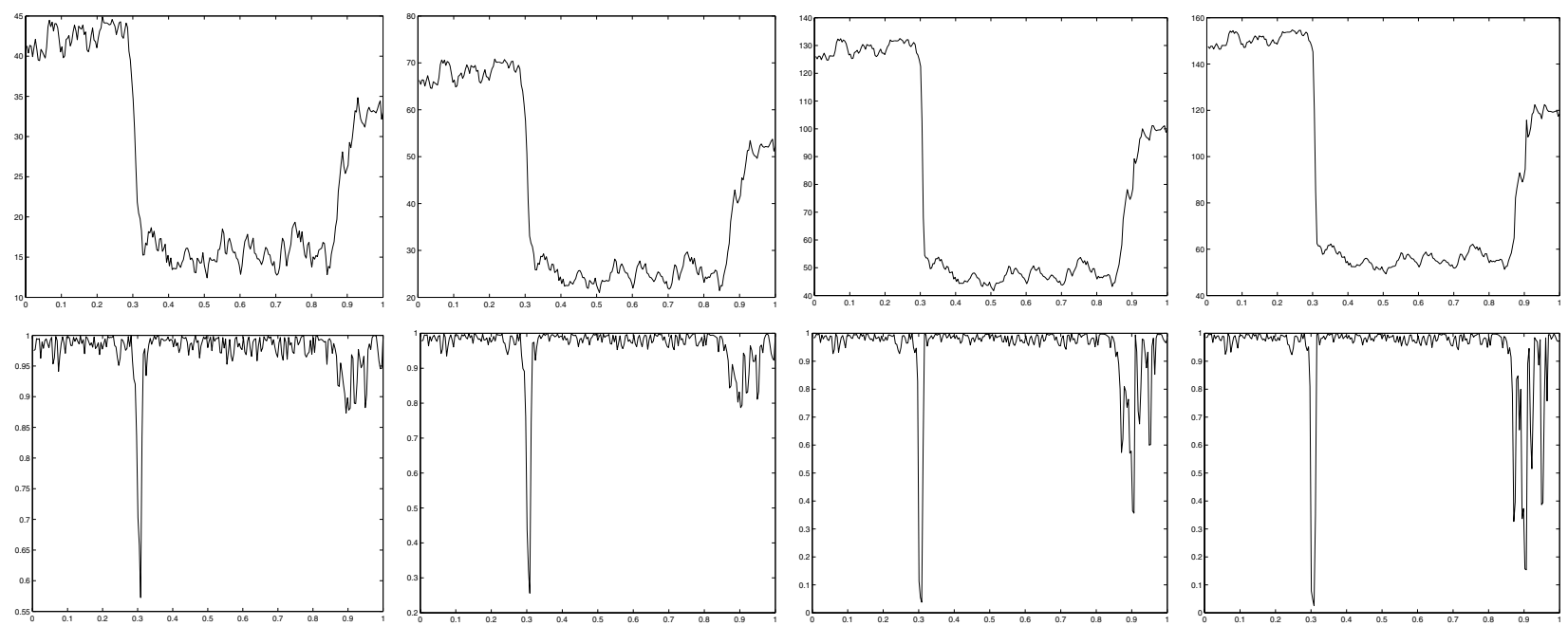

Figure 6. Cross sections at $x=60$ of the snapshots of Figure 5: $\left\{u_{h}^{m}\right\}$ (top row) and $\left\{\varphi_{h}^{m}\right\}$ (bottom row).

Test 3. This test studies the evolution of the flow starting with the initial data $u_{0} \equiv 0$ and $\varphi_{0} \equiv 0.5$. Also, we use a larger fidelity constant $\gamma=5$ for a better resolution, but keep all other parameters be same as in Tests 1 and 2 .

Figure 5 displays the computed soft image $u_{h}^{m}$ (top row) and edge function $\varphi_{h}^{m}$ (bottom row) at time steps $t_{5}, t_{9}, t_{27}$, and $t_{49}$. Figure 6 shows the cross section at $x=60$ of each $u_{h}^{m}$ and $\varphi_{h}^{m}$ of Figure 5. The simulation shows that the edges are detected according to contrast (see the bottom rows of Figs. 5 and 6 ), since as the intensity slowly grows in time, the (detected) edges get sharpened while the contrast improves. Again, we obtain a satisfactorily recovered image in the limit (as $t$ becomes large), but the simulation time is significantly longer (by a factor of 3) than those of Tests 1 and 2 . 
Acknowledgements. The authors would like to thank the Mathematisches Forschungsinstitut Oberwolfach for the kind hospitality and opportunity of its "Research in Pairs" program. They are also grateful to J. Vogelgesang (ETH Zürich) for providing the numerical tests.

\section{REFERENCES}

[1] R.A. Adams, Sobolev Spaces. Academic Press, New York (1975).

[2] L. Ambrosio and V.M. Tortorelli, Approximation of functionals depending on jumps by elliptic functionals via $\Gamma$-convergence. Comm. Pure Appl. Math. 43 (1990) 999-1036.

[3] L. Ambrosio and V.M. Tortorelli, On the approximation of functionals depending on jumps by quadratic, elliptic functionals. Boll. Un. Mat. Ital. 6-B (1992) 105-123.

[4] L. Ambrosio, N. Fusco and D. Pallara, Functions of Bounded Variation and Free Discontinuity Problems. Oxford University Press (2000).

[5] J.W. Barrett, X. Feng and A. Prohl, Convergence of a fully discrete finite element method for a degenerate parabolic system modeling nematic liquid crystals with variable degree of orientation, preprint.

[6] G. Bellettini and A. Coscia, Discrete approximation of a free discontinuity problem. Numer. Funct. Anal. Optimiz. 15 (1994) 201-224.

[7] A. Blake and A. Zisserman, Visual reconstruction. MIT Press, Cambridge, MA (1987).

[8] B. Bourdin, Image segmentation with a finite element method. ESAIM: M2AN 33 (1999) 229-244.

[9] A. Braides, Approximation of free-discontinuity problems. Lect. Notes Math. 1694, Springer-Verlag (1998).

[10] A. Braides and G. Dal Maso, Nonlocal approximation of the Mumford-Shah functional. Calc. Var. Partial Differential Equations 5 (1997) 293-322.

[11] S.C. Brenner and L.R. Scott, The Mathematical Theory of Finite Element Methods, Second Edition, Springer-Verlag, New York (2001).

[12] J.W. Cahn and J.E. Hilliard, Free energy of a nonuniform system I, Interfacial free energy. J. Chem. Phys. 28 (1958) $258-267$.

[13] A. Chambolle, Image segmentation by variational methods: Mumford-Shah functional and the discrete approximation. SIAM J. Appl. Math. 55 (1995) 827-863.

[14] A. Chambolle and G. Dal Maso, Discrete approximation of the Mumford-Shah functional in dimension two. ESAIM: M2AN 33 (1999) 651-672.

[15] P.G. Ciarlet, Basic error estimates for elliptic problems, in Handbook of Numer. Anal. II, Elsevier Sciences Publishers (1991).

[16] G. Dal Maso, An introduction to $\Gamma$-convergence, Birkhäuser Boston, Boston, MA (1993).

[17] E. De Giorgi, M. Carriero and A. Leaci, Existence theorem for a minimum problem with discontinuity set. Arch. Rat. Mech. Anal. 108 (1989) 195-218.

[18] E. De Giorgi and T. Franzoni, Su un tipo di convergenza variazionale. Atti Accad. Naz. Lincei Rend. Cl. Sci. Fis. Mat. Natur. 58 (1975) 842-850.

[19] F. Dibos and E. Séré, An approximation result for the minimizers of the Mumford-Shah functional. Boll. Un. Mat. Ital. A 11 (1997).

[20] C.M. Elliott, D.A. French and F.A. Milner, A second order splitting method for the Cahn-Hilliard equation. Numer. Math. 54 (1989) $575-590$

[21] S. Esedoglu and J. Shen, Digital inpainting based on the Mumford-Shah-Euler image model. European J. Appl. Math. 13 (2002) 353-370.

[22] X. Feng and A. Prohl, Analysis of total variation flow and its finite element approximations. ESAIM: M2AN 37 (2003) 533-556.

[23] X. Feng and A. Prohl, On gradient flow of the Mumford-Shah functional. (in preparation).

[24] D. Geman and S. Geman, Stochastic relaxation, Gibbs distributions and the Bayesian restoration of images. IEEE Trans. Patten Anal. Mach. Intell. 6 (1984) 721-741.

[25] R. Glowinski, J.L. Lions and R. Trémoliéres, Numerical analysis of variational inequalities. North-Holland, New York. Stud. Math. Appl. 8 (1981).

[26] M. Gobbino, Gradient flow for the one-dimensional Mumford-Shah strategies. Ann. Scuola Norm. Sup. Pisa Cl. Sci. 27 (1998) 145-193.

[27] J.L. Lions, Quelques méthodes de résolution des problèmes aux limites non linéaires, Dunod (1969).

[28] R. March and M. Dozio, A variational method for the recovery of smooth boundaries. Im. Vis. Comp. 15 (1997) 705-712.

[29] L. Modica, The gradient theory of phase transitions and the minimal interface criterion. Arch. Rational Mech. Anal. 98 (1987) $123-142$.

[30] L. Modica and S. Mortola, Un esempio di Г-convergenza. Boll. Un. Mat. Ital. B 14 (1977) 285-299.

[31] J.-M. Morel and S. Solimini, Variational Methods in Image Segmentation, Birkhäuser (1995).

[32] D. Mumford and J. Shah, Optimal approximations by piecewise smooth functions and associated variational problems. Comm. Pure Appl. Math. 42 (1989) 577-685.

[33] R.H. Nochetto and C. Verdi, Convergence past singularities for a fully discrete approximation of curvature-driven interfaces. SIAM J. Numer. Anal. 34 (1997) 490-512.

[34] J. Simon, Compact sets in the space $L^{p}(0, T ; B)$. Ann. Mat. Pura Appl. 146 (1987) 65-96.

[35] M. Struwe, Geometric evolution problems. IAS/Park City Math. Series 2 (1996) 259-339. 